\title{
The Extended Relativity Theory in Clifford Phase Spaces and Modifications of Gravity at the Planck/Hubble scales
}

\author{
Carlos Castro \\ Center for Theoretical Studies of Physical Systems \\ Clark Atlanta University, Atlanta, Georgia. 30314, perelmanc@hotmail.com
}

February 2013, Revised April 2013

\begin{abstract}
We extend the construction of Born's Reciprocal Phase Space Relativity to the case of Clifford Spaces which involve the use of polyvectors and a lower/upper length scale. We present the generalized polyvector-valued velocity and acceleration/force boosts in Clifford Phase Spaces and find an explicit Clifford algebraic realization of the velocity and acceleration/force boosts. Finally, we provide a Clifford Phase-Space Gravitational Theory based in gauging the generalization of the Quaplectic group and invoking Born's reciprocity principle between coordinates and momenta (maximal speed of light velocity and maximal force). The generalized gravitational vacuum field equations are explicitly displayed. We conclude with a brief discussion on the role of higher-order Finsler geometry in the construction of extended relativity theories with an upper and lower bound to the higher order accelerations (associated with the higher order tangent and cotangent spaces). We explain how to find the procedure that will allow us to find the $n$-ary analog of the Quaplectic group transformations which will now mix the $X, P, Q, \ldots \ldots$ coordinates of the higher order tangent (cotangent) spaces in this extended relativity theory based on Born's reciprocal gravity and $n$-ary algebraic structures.
\end{abstract}

\section{Introduction : Born's Reciprocal Relativity in Phase Spaces}

In recent years we have argued that the underlying fundamental physical principle behind string theory, not unlike the principle of equivalence and general covariance in Einstein's general relativity, might well be related to the existence of an invariant minimal length scale (Planck scale) attainable in nature. A scale relativistic theory involving spacetime resolutions was developed long ago by 
Nottale where the Planck scale was postulated as the minimum observer independent invariant resolution in Nature [1]. Since "points" cannot be observed physically with an ultimate resolution, they are fuzzy and smeared out into fuzzy balls of Planck radius of arbitrary dimension. For this reason one must construct a theory that includes all dimensions (and signatures) on the equal footing. Because the notion of dimension is a topological invariant, and the concept of a fixed dimension is lost due to the fuzzy nature of points, dimensions are resolution-dependent, one must also include a theory with all topologies as well. It turned out that Clifford algebras contained the appropriate algebrogeometric features to implement this principle of polydimensional transformations that reshuffle a five-brane history for a membrane history, for example. For an extensive review of this Extended Relativity Theory in Clifford Spaces that encompasses the unified dynamics of all p-branes, for different values of the dimensions of the extended objects, and numerous physical consequences, see $[2],[4],[3],[6]$.

An upper limit on the maximal acceleration of particles was proposed long ago by Cainello [9]. This idea is a direct consequence of a suggestion made years earlier by Max Born on a Reciprocal Relativity principle operating in Phase Spaces [8], [10] where there is an upper bound on the four-force (maximal string tension or tidal forces in strings ) acting on a particle as well as an upper bound in the particle's velocity given by the speed of light.

In this introductory section we briefly review Born's Reciprocal Phase Space Relativity and the principle of Maximal-acceleration/force Relativity from the perspective of $8 D$ Phase Spaces and the $U(1,3)$ Group. The $U(1,3)=S U(1,3) \otimes$ $U(1)$ Group transformations which leave invariant the phase-space intervals under rotations, velocity and acceleration boosts, were found by Low [10] and can be simplified drastically when the velocity/acceleration boosts are taken to lie in the $z$-direction, leaving the transverse directions $x, y, p_{x}, p_{y}$ intact ; i.e., the $U(1,1)=S U(1,1) \otimes U(1)$ subgroup transformations leave invariant the phasespace interval given by (in units of $\hbar=c=1$ )

$$
\begin{gathered}
(d \omega)^{2}=(d T)^{2}-(d X)^{2}+\frac{(d E)^{2}-(d P)^{2}}{b^{2}}= \\
(d \tau)^{2}\left[1+\frac{(d E / d \tau)^{2}-(d P / d \tau)^{2}}{b^{2}}\right]=(d \tau)^{2}\left[1-\frac{m^{2} g^{2}(\tau)}{m_{P}^{2} A_{\max }^{2}}\right] .
\end{gathered}
$$

where we have factored out the proper time infinitesimal $(d \tau)^{2}=d T^{2}-d X^{2}$ in eq-(1.1) and the maximal proper-force is set to be $b \equiv m_{P} A_{\max } \cdot m_{P}$ is the Planck mass $1 / L_{P}$ so that $b=\left(1 / L_{P}\right)^{2}$, may also be interpreted as the maximal string tension when $L_{P}$ is the Planck scale.

The quantity $g(\tau)$ is the proper four-acceleration of a particle of mass $m$ in the $z$-direction which we take to be defined by the $X$ coordinate. The interval $(d \omega)^{2}$ described by Low [10] is $U(1,3)$-invariant for the most general transformations in the $8 D$ phase-space. These transformations are rather elaborate, so we refer to the references [10] for details. 
The appearance of the $U(1,3)$ group in $8 D$ Phase Space is not too surprising since it could be seen as the " complex doubling " version of the Lorentz group $S O(1,3)$. Low discussed the irreducible unitary representations of such $U(1,3)$ group and the relevance for the strong interactions of quarks and hadrons since $U(1,3)$, with 16 generators, contains the $S U(3)$ group.

The analog of the Lorentz relativistic factor in eq-(1.1) involves the ratios of two proper forces. One variable force is given by $m g(\tau)$ and the maximal proper force sustained by an elementary particle of mass $m_{P}$ is assumed to be $F_{\max }=m_{\text {Planck }} c^{2} / L_{P}$. When $m=m_{P}$, the ratio-squared of the forces appearing in the relativistic factor of eq-(1.1) becomes then $g^{2} / A_{\text {max }}^{2}$, and the phase space interval coincides with the geometric interval discussed by

The transformations laws of the coordinates in that leave invariant the interval (1.1) were given by [10]:

$$
\begin{aligned}
& T^{\prime}=T \cosh \xi+\left(\frac{\xi_{v} X}{c^{2}}+\frac{\xi_{a} P}{b^{2}}\right) \frac{\sinh \xi}{\xi} . \\
& E^{\prime}=E \cosh \xi+\left(-\xi_{a} X+\xi_{v} P\right) \frac{\sinh \xi}{\xi} . \\
& X^{\prime}=X \cosh \xi+\left(\xi_{v} T-\frac{\xi_{a} E}{b^{2}}\right) \frac{\sinh \xi}{\xi} . \\
& P^{\prime}=P \cosh \xi+\left(\frac{\xi_{v} E}{c^{2}}+\xi_{a} T\right) \frac{\sinh \xi}{\xi} .
\end{aligned}
$$

The $\xi_{v}$ is velocity-boost rapidity parameter and the $\xi_{a}$ is the force/accelerationboost rapidity parameter of the primed-reference frame. They are defined respectively :

$$
\tanh \left(\frac{\xi_{v}}{c}\right)=\frac{v}{c} . \quad \tanh \left(\frac{\xi_{a}}{b}\right)=\frac{m a}{m_{P} A_{\max }} .
$$

The effective boost parameter $\xi$ of the $U(1,1)$ subgroup transformations appearing in eqs-(2-2a, 2-2d) is defined in terms of the velocity and acceleration boosts parameters $\xi_{v}, \xi_{a}$ respectively as:

$$
\xi \equiv \sqrt{\frac{\xi_{v}^{2}}{c^{2}}+\frac{\xi_{a}^{2}}{b^{2}}} .
$$

Straightforward algebra allows us to verify that these transformations leave the interval of eq- (1.1) in classical phase space invariant. They are are fully consistent with Born's duality Relativity symmetry principle [8] $(Q, P) \rightarrow(P,-Q)$. By inspection we can see that under Born reciprocity, the transformations in eqs-(1.2a-1.2d) are rotated into each other, up to numerical $b$ factors in order to match units. When on sets $\xi_{a}=0$ in $(1.2 \mathrm{a}-1.2 \mathrm{~d})$ one recovers automatically the standard Lorentz transformations for the $X, T$ and $E, P$ variables separately, leaving invariant the intervals $d T^{2}-d X^{2}=(d \tau)^{2}$ and $\left(d E^{2}-d P^{2}\right) / b^{2}$ separately.

Also the transformations leave invariant the symplectic two-form 


$$
d T^{\prime} \wedge d E^{\prime}-d X^{\prime} \wedge d P^{\prime}=d T \wedge d E-d X \wedge d P
$$

For simplicity, unless otherwise indicated, we shall choose the natural units $\hbar=c=G=1$ so that $b=m_{P}=L_{P}=1$. The composition of two successive transformations like in eqs-(1.2) yields a similar transformation as (1.2) due to the group nature of $U(1,1)$. The composition of the rapidity parameters is simplified enormously as

$$
\xi_{v}^{\prime \prime}=\xi_{v}^{\prime}+\xi_{v} ; \quad \xi_{a}^{\prime \prime}=\xi_{a}^{\prime}+\xi_{a} ; \quad \xi^{\prime \prime}=\xi^{\prime}+\xi
$$

and obeying the relations

$$
(\xi)^{2}=\left(\xi_{v}\right)^{2}+\left(\xi_{a}\right)^{2} ; \quad\left(\xi^{\prime}\right)^{2}=\left(\xi_{v}^{\prime}\right)^{2}+\left(\xi_{a}^{\prime}\right)^{2} ; \quad\left(\xi^{\prime \prime}\right)^{2}=\left(\xi_{v}^{\prime \prime}\right)^{2}+\left(\xi_{a}^{\prime \prime}\right)^{2}
$$

when the following conditions are obeyed

$$
\begin{aligned}
& \frac{\xi_{a}}{\xi}=\frac{\xi_{a}^{\prime}}{\xi^{\prime}}=\frac{\xi_{a}^{\prime \prime}}{\xi^{\prime \prime}}=\frac{\xi_{a}^{\prime}+\xi_{a}}{\xi^{\prime}+\xi} \\
& \frac{\xi_{v}}{\xi}=\frac{\xi_{v}^{\prime}}{\xi^{\prime}}=\frac{\xi_{v}^{\prime \prime}}{\xi^{\prime \prime}}=\frac{\xi_{v}^{\prime}+\xi_{v}}{\xi^{\prime}+\xi}
\end{aligned}
$$

from the relations (1.6-1.8) one can show that

$$
\left(\xi^{\prime \prime}\right)^{2}=\left(\xi_{v}^{\prime \prime}\right)^{2}+\left(\xi_{a}^{\prime \prime}\right)^{2}=\left(\xi_{v}^{\prime}+\xi_{v}\right)^{2}+\left(\xi_{a}^{\prime}+\xi_{a}\right)^{2}=\left(\xi^{\prime}+\xi\right)^{2}
$$

The Eddington-Dirac large numbers coincidence ( and an ultraviolet/infrared entanglement ) can be easily implemented if one equates the upper bound on the proper-four force sustained by a fundamental particle, $(m g)_{\text {bound }}=m_{P}\left(c^{2} / L_{P}\right)$, with the proper-four force associated with the mass of the (observed) universe $M_{U}$, and whose minimal acceleration $c^{2} / R$ is given in terms of an infrared-cutoff $R$ ( the Hubble horizon radius ). Equating these proper-four forces gives

$$
\frac{m_{P} c^{2}}{L_{P}}=\frac{M_{U} c^{2}}{R} \Rightarrow \frac{M_{U}}{m_{P}}=\frac{R}{L_{P}} \sim 10^{61} .
$$

from this equality of proper-four forces associated with a maximal $/$ minimal acceleration one infers $M_{U} \sim 10^{61} m_{\text {Planck }} \sim 10^{61} 10^{19} m_{\text {proton }}=10^{80} m_{\text {proton }}$ which agrees with observations and with the Eddington-Dirac number $10^{80}[3]$

$$
N=10^{80}=\left(10^{40}\right)^{2} \sim\left(\frac{F_{e}}{F_{G}}\right)^{2} \sim\left(\frac{R}{r_{e}}\right)^{2} .
$$

where $F_{e}=e^{2} / r^{2}$ is the electrostatic force between an electron and a proton ; $F_{G}=G m_{e} m_{\text {proton }} / r^{2}$ is the corresponding gravitational force and $r_{e}=e^{2} / m_{e} \sim$ $10^{-13} \mathrm{~cm}$ is the classical electron radius (in units $\hbar=c=1$ ).

One may notice that the above equation (1.10) is also consistent with the Machian postulate [3] that the rest mass of a particle is determined via the gravitational potential energy due to the other masses in the universe. In particular, by equating 


$$
m_{i} c^{2}=G m_{i} \sum_{j} \frac{m_{j}}{\left|r_{i}-r_{j}\right|}=\frac{G m_{i} M_{U}}{R} \Rightarrow \frac{c^{2}}{G}=\frac{M_{U}}{R} .
$$

Due to the negative binding energy, the composite mass $m_{12}$ of a system of two objects of mass $m_{1}, m_{2}$ is not equal to the sum $m_{1}+m_{2}>m_{12}$. We can now arrive at the conclusion that the minimal acceleration $c^{2} / R$ is also the same acceleration induced on a test particle of mass $m$ by a spherical mass distribution $M_{U}$ inside a radius $R$. The acceleration felt by a test particle of mass $m$ sitting at the edge of the observable Universe ( at the Hubble horizon radius $R$ ) is

$$
|a|=\frac{G M_{U}}{R^{2}}
$$

From the last two equations one gets the same expression for the minimal acceleration

$$
a=a_{\text {minimal }}=\frac{c^{2}}{R} .
$$

which is of the same order of magnitude as the anomalous acceleration of the Pioneer and Galileo spacecrafts $a \sim 10^{-8} \mathrm{~cm} / \mathrm{s}^{2}$.

Let us examine closer the equality between the proper-four forces

$$
\frac{m_{P} c^{2}}{L_{P}}=\frac{M_{U} c^{2}}{R} \Rightarrow \frac{m_{P}}{L_{P}}=\frac{M_{U}}{R}=\frac{c^{2}}{G} .
$$

The last term in eq-(1.15) is directly obtained after implementing the Machian principle. Thus, one concludes from eq-(1.15 ) that as the universe evolves in time one must have the conserved ratio of the quantities $M_{U} / R=c^{2} / G=$ $m_{P} / L_{P}$. This interesting possibility, advocated by Dirac long ago, for the fundamental constants $\hbar, c, G, \ldots$. to vary over cosmological time is a plausible idea with the provision that the above ratios satisfy the relations in eq-(1.15) at any given moment of cosmological time. If the fundamental constants do not vary over time then the ratio $M_{U} / R=c^{2} / G$ must refer then to the asymptotic values of the Hubble horizon radius $R=R_{\text {asymptotic }}$.

The outline of the rest of this work goes as follows. In section 2.1 we extend the construction of Born's Reciprocal Phase Space Relativity to the case of Clifford Spaces which involve the use of polyvectors and a lower and upper length scale. In particular we explain how to build a generalized gravitational theory invoking Born's reciprocity principle. In $\mathbf{2 . 2}$ we display the generalized polyvector-valued velocity and acceleration/force boosts in Clifford Phase Spaces. In 2.3 we construct an explicit Clifford algebraic realization of the velocity and acceleration/force boosts furnishing the same transformations as in eqs-(1.2), and show how to generalize and extend these transformations to the full-fledge Clifford Phase Space involving polyvector-valued coordinates and momenta. 
In section 3 we construct a Clifford Phase Space Gravitational Theory based in gauging the Clifford generalization of the Quaplectic group. The generalized gravitational vacuum field equations are explicitly displayed. Finally, in section 4 we present our conclusions with a brief discussion of the role of Finsler geometry in the construction of extended relativity theories with an upper and lower bound to the higher order accelerations associated with the higher order tangent and cotangent spaces.

\section{The Extended Relativity in Born-Clifford Phase Spaces}

\subsection{The Clifford-Phase Space with a Lower and Upper Length scales}

We briefly embark into the extended relativity theory in Clifford ( $C$-spaces), [2], [3], [11] by generalizing the notion of a spacetime interval in Minkwoski space to C-space

$$
d X^{2}=d \sigma^{2}+d x_{\mu} d x^{\mu}+d x_{\mu \nu} d x^{\mu \nu}+d x_{\mu \nu \rho} d x^{\mu \nu \rho}+\ldots
$$

The Clifford valued polyvector

$X=X^{M} E_{M}=\sigma \mathbf{1}+x^{\mu} \gamma_{\mu}+x^{\mu \nu} \gamma_{\mu} \wedge \gamma_{\nu}+\ldots . x^{\mu_{1} \mu_{2} \ldots \mu_{D}} \gamma_{\mu_{1}} \wedge \gamma_{\mu_{2}} \ldots \wedge \gamma_{\mu_{D}}$.

denotes the position of a polyparticle in a manifold, called Clifford space or $C$-space. The series of terms in (2.1) terminates at a finite value depending on the dimension $D$. A real Clifford algebra $C l(p, q)$ with $p+q=D$ has $2^{D}$ basis elements. The gammas $\gamma^{\mu}$ correspond to a Clifford algebra associated with a flat spacetime

$$
\left\{\gamma^{\mu}, \gamma^{\nu}\right\}=2 \eta^{\mu \nu}
$$

but in general one could extend this formulation to curved spacetimes with a metric $g^{\mu \nu}$ by introducing the vielbeins $\gamma_{\mu}=e_{\mu}^{a} \gamma_{a}$.

The connection to strings and p-branes can be seen as follows. In the case of a closed string (a 1-loop) embedded in a target flat spacetime background of $D$ dimensions, one represents the projections of the closed string (1-loop) onto the embedding spacetime coordinate-planes by the variables $x_{\mu \nu}$. These variables represent the respective areas enclosed by the projections of the closed string (1-loop) onto the corresponding embedding spacetime planes. Similarly, one can embed a closed membrane (a 2-loop) onto a $D$-dim flat spacetime, where the projections given by the antisymmetric variables $x_{\mu \nu \rho}$ represent the corresponding volumes enclosed by the projections of the 2-loop along the hyperplanes of the flat target spacetime background. 
This procedure can be carried to all closed p-branes ( p-loops ) where the values of $\mathrm{p}$ are $p=0,1,2,3, \ldots . D-2$. The $p=0$ value represents the center of mass and the coordinates $x^{\mu \nu}, x^{\mu \nu \rho} \ldots$ have been coined in the string-brane literature [7] as the holographic areas, volumes, ...projections of the $p$-loops ( closed p-branes ) onto the embedding spacetime coordinate planes/hyperplanes.

If we take the differential $d X$ and compute the scalar product among two polyvectors $<d \tilde{X} d X>_{\text {scalar }}$ we obtain the C-space extension of the particle's proper time in Minkwoski space. The symbol $\tilde{X}$ denotes the rever sion operation and involves reversing the order of all the basis $\gamma^{\mu}$ elements in the expansion of $X$. It is the analog of the transpose ( Hermitian ) conjugation. The C-space proper time associated with a polyparticle motion is then

$$
d \Sigma^{2}=(d \sigma)^{2}+L_{P}^{2 D-2} d x_{\mu} d x^{\mu}+\mathrm{E}_{P}^{2 D-4} d x_{\mu \nu} d x^{\mu \nu}+\ldots \ldots
$$

Here we have explicitly introduced the Planck scale $\mathrm{L}_{P}$ since a length parameter is needed in order to tie objects of different dimensionality together: 0-loops, 1-loops,..., $p$-loops. Einstein introduced the speed of light as a universal absolute invariant in order to "unite" space with time (to match units) in the Minkwoski space interval:

$$
d s^{2}=c^{2} d t^{2}-d x_{i} d x^{i}
$$

A similar unification is needed here to "unite" objects of different dimensions, such as $x^{\mu}, x^{\mu \nu}$, etc... The Planck scale then emerges as another universal invariant in constructing an extended scale relativity theory in C-spaces.

To continue along the same path, we consider the analog of Lorentz transformations in C-spaces which transform a poly-vector $X$ into another poly-vector $X^{\prime}$ given by $X^{\prime}=R X R^{-1}$ with

$$
\mathbf{R}=e^{\theta^{M} E_{M}}=\exp \left[\theta \mathbf{1}+\theta^{\mu} \gamma_{\mu}+\theta^{\mu_{1} \mu_{2}} \gamma_{\mu_{1}} \wedge \gamma_{\mu_{2}} \ldots . .\right] .
$$

and

$$
\mathbf{R}^{-1}=e^{-\theta^{M} E_{M}}=\exp \left[-\left(\theta \mathbf{1}+\theta^{\nu} \gamma_{\nu}+\theta^{\nu_{1} \nu_{2}} \gamma_{\nu_{1}} \wedge \gamma_{\nu_{2}}+\ldots . .\right)\right]
$$

where the theta parameters $\theta ; \theta^{\mu} ; \theta^{\mu \nu}, \ldots$. in $(2.5,2.6)$ are the components of the Clifford-valued parameter $\Theta=\theta^{M} E_{A} M$. The transformations $X^{\prime}=R X R^{-1}$ are the $\mathrm{C}$-space version of the Lorentz rotations/boosts parameters. If one requires to satisfy the condition $\mathbf{R}^{-1}=\tilde{\mathbf{R}}$, after using the tilde operation (which reverses the order of the products of the gamma basis generators present in the $E_{M}$ polyvectors ) so that the inner product $\langle\tilde{X} X\rangle$ (the bracket operation denotes taking the scalar part of the Clifford geometric product) is invariant under polyrotations, one is forced to restrict the grades of the polyvector elements allowed in the definition of $\mathbf{R}$. Hence, the condition $\mathbf{R}^{-1}=\tilde{\mathbf{R}}$ will restrict the 
type of terms allowed inside the exponential defining the rotor $R$ because the reversal operation (denoted below by $\dagger$ ) of a $p$-vector obeys

$$
\left(\gamma_{\mu_{1}} \wedge \gamma_{\mu_{2}} \ldots . \wedge \gamma_{\mu_{p}}\right)^{\dagger}=\gamma_{\mu_{p}} \wedge \gamma_{\mu_{p-1}} \ldots . \wedge \gamma_{\mu_{2}} \wedge \gamma_{\mu_{1}}=(-1)^{p(p-1) / 2} \gamma_{\mu_{1}} \wedge \gamma_{\mu_{2}} \ldots . . \wedge \gamma_{\mu_{p}}
$$

Hence only those terms that change sign (under the reversal operation) are permitted inside the exponential defining $\mathbf{R}=\exp \left[\theta^{M} E_{M}\right]$.

Our task now is to construct a Relativity theory that implements simultaneously the Born's reciprocity principle (associated with a minimal and maximal acceleration/scale) . The upper scale $R$ can be set to the Hubble scale which can be seen as infrared regulator. The minimal scale is set equal to the Planck length $L_{P}$ which can be seen as an ultraviolet regulator.

The interval in Clifford-Phase-Spaces is defined

$$
\begin{gathered}
d \Sigma^{2}=<d \tilde{X} d X>+\frac{1}{\mathcal{F}^{2}}<d \tilde{P} d P>= \\
\left(\frac{d \sigma}{L_{P}^{D-1}}\right)^{2}+d x_{\mu} d x^{\mu}+\frac{d x_{\mu \nu} d x^{\mu \nu}}{L_{P}^{2}}+\frac{d x_{\mu \nu \rho} d x^{\mu \nu \rho}}{L_{P}^{4}}+\ldots+ \\
\frac{1}{\mathcal{F}^{2}}\left[\frac{(d \rho)^{2}}{(\hbar / R)^{2 D-2}}+d p_{\mu} d p^{\mu}+\frac{d p_{\mu \nu} d p^{\mu \nu}}{(\hbar / R)^{2}}+\frac{d p_{\mu \nu \rho} d p^{\mu \nu \rho}}{(\hbar / R)^{4}}+\ldots . .\right]
\end{gathered}
$$

where the maximal force is

$$
\mathcal{F}=\frac{m_{P} c^{2}}{L_{P}}=\frac{M_{U} c^{2}}{R}=\frac{c^{4}}{G}
$$

The infrared scale $R \rightarrow \infty$ limit is set in conjunction with the vanishing limit of the holographic momenta variables (to avoid singularities)

$$
p^{\mu \nu} \rightarrow 0, p^{\mu \nu \rho} \rightarrow 0, \ldots
$$

constraining the interval in the Born-Clifford Phase Space to be of the form

$$
\begin{gathered}
d \Sigma^{2} \Rightarrow \frac{(d \sigma)^{2}}{L_{P}^{2 D-2}}+d x_{\mu_{1}} d x^{\mu_{1}}+\frac{d x_{\mu_{1} \mu_{2}} d x^{\mu_{1} \mu_{2}}}{L_{P}^{2}}+\frac{d x_{\mu_{1} \mu_{2} \mu_{3}} d x^{\mu_{1} \mu_{2} \mu_{3}}}{L_{P}^{4}}+\ldots+ \\
\frac{d x_{\mu_{1} \mu_{2} \mu_{3} \ldots \mu_{D-1}} d x^{\mu_{1} \mu_{2} \mu_{3} \ldots \mu_{D-1}}}{L_{P}^{2 D-2}}+\frac{1}{\mathcal{F}^{2}} d p_{\mu} d p^{\mu} .
\end{gathered}
$$

In the classical limit $\hbar \rightarrow 0$ the Planck scale $L_{P}=\sqrt{\left(\hbar G / c^{3}\right)} \rightarrow 0$. Thus, the ultraviolet limit $L_{P} \rightarrow 0$ is accompanied with the vanishing limit of the holographic coordinate variables

$$
x^{\mu \nu} \rightarrow 0, x^{\mu \nu \rho} \rightarrow 0, \ldots
$$

and the interval reduces further to the standard Born Phase Space interval

$$
d \Sigma^{2} \Rightarrow d x_{\mu} d x^{\mu}+\frac{1}{\mathcal{F}^{2}} d p_{\mu} d p^{\mu}=
$$




$$
\begin{gathered}
d \tau^{2}\left[1+\frac{\left(d p_{\mu} / d \tau\right)\left(d p^{\mu} / d \tau\right)}{\mathcal{F}^{2}}\right]= \\
d \tau^{2}\left[1+\frac{1}{\mathcal{F}^{2}} m^{2} \frac{d^{2} x_{\mu}}{d \tau^{2}} \frac{d^{2} x^{\mu}}{d \tau^{2}}\right]=d \tau^{2}\left[1-\frac{m^{2} g^{2}(\tau)}{\mathcal{F}^{2}}\right] .
\end{gathered}
$$

When $\mathcal{F} \rightarrow \infty$ the Phase Space interval (2.11) reduces further to the Minkowski spacetime interval.

As stated above, when $\hbar \rightarrow 0$ one has that the Planck scale $L_{P} \rightarrow 0$ and also one should notice that

$$
\begin{gathered}
m_{P}=\frac{\hbar}{c L_{P}}=\frac{\hbar}{c \sqrt{\left(\hbar G / c^{3}\right)}} \rightarrow 0 . \quad a_{\max }=\frac{c^{2}}{L_{P}}=\sqrt{\left(c^{7} / \hbar G\right)} \rightarrow \infty \Rightarrow \\
m_{P} a_{\max }=\frac{c^{4}}{G}=\mathcal{F} .
\end{gathered}
$$

Despite that the maximal acceleration is infinite this does not necessarily imply that the maximal force is also divergent due to the fact that the $m_{P} \rightarrow 0$. This type of limit in (2.12) has also been studied by [15] pertaining to the notion of "relativity of locality" in curved phase space.

Hence there are two regimes that interpolate from the Born-Clifford Phase Space interval to the standard point-particle Born Phase Space interval (related to the existence of a maximal force). In the infinite distance ( infrared ) limit $R \rightarrow \infty$ the minimum momentum collapses to zero and in order to avoid singularities we must set all the holographic momenta to zero that leads to the decoupling of the holographic momenta variables. In the classical limit $\hbar \rightarrow 0$ the Planck scale collapses to zero and in order to avoid singularities we must set all the holographic coordinate variables to zero so one ends up solely with the Born's Reciprocal Phase Space Relativity interval associated with a point particle subjected to an upper bound on the force $\mathcal{F}=c^{4} / G$ and with an upper bound on the speed given by $c$.

Born's Reciprocal Relativity theory in Phase Spaces [19] leads to modified dispersion relations involving both coordinates and momenta, and whose truncations furnish Lorentz-violating dispersion relations which appear in Finsler Geometry, rainbow-metrics models and Double (deformed) Special Relativity [14]. We provided in [18] six specific results stemming from Born's reciprocal Relativity and which are not present in Special Relativity. These were : momentum-dependent time delay in the emission and detection of photons; energy-dependent notion of locality; superluminal behavior; relative rotation of photon trajectories due to the aberration of light; invariance of areas-cells in phase-space and modified dispersion relations. One of the most interesting conclusions was that there are null hypersurfaces in a flat phase-spaces where points can have superluminal $v>c$ behavior in ordinary spacetime, despite corresponding to a null hypersurface in a flat phase-space. Superluminal behavior in spacetime can occur without having superluminal behavior in $C$-spaces [5].

Gravity in curved phase-spaces and two-times Physics following the strict formalism of Lagrange-Finsler and Hamilton-Cartan geometry was analyzed in 
[19]. The scalar curvature of the $8 D$ cotangent bundle (phase space) was explicitly evaluated and a generalized gravitational action in $8 D$ was constructed that yields the observed value of the cosmological constant and the Brans-DickeJordan Gravity action in $4 D$ as two special cases. It was found that the geometry of the momentum space can be linked to the observed value of the cosmological constant when the curvature in momentum space is very large, namely the small size of $P$ is of the order of $\left(1 / R_{\text {Hubble }}\right)$. More general $8 D$ actions can be developed from Finsler geometric methods that involve sums of 5 distinct types of torsion squared terms and 3 distinct curvature scalars.

A Born's reciprocal complex gravitational theory as a local gauge theory in $8 D$ of the deformed Quaplectic group was developed by [17]. The gauge group was given by the semi-direct product of $U(1,3)$ with the deformed (noncommutative) Weyl-Heisenberg group involving four noncommutative coordinates and momenta. The (deformed) Quaplectic group acts as the automorphism group along the internal fiber coordinates. One must not confuse the deformed complex gravity constructed in [17] with the noncommutative gravity work in the literature. Another salient feature of [17] was that the metric is complex with symmetric real components and antisymmetric imaginary ones. An action in $8 D$ involving 2 curvature scalars and torsion-squared terms was presented. In section 3 we shall construct a Clifford Phase Space Gravitational Theory based in gauging the polyvector generalization of the Quaplectic group. Before doing so we will continue with studying the transformations of polyvector-valued coordinates and momenta in Clifford Phase Spaces.

\subsection{Generalized Velocity and Acceleration Boosts in Clif- ford Phase Spaces}

The most general transformations in C-phase-space mix different grade components of the polyvectors $X^{M}, P^{M}$. To begin with we shall study those transformations which do not mix the different grade components and we shall choose the natural units $\hbar=c=G=1$ so that $b=m_{P}=L_{P}=1$. The pure areal-boost transformations of the areal coordinates and momenta, involving the areal-velocity $\xi_{v}^{12}$ and areal-acceleration rapidity boosts $\xi_{a}^{12}$ parameters , are given by

$$
\begin{aligned}
& X^{\prime 01}=X^{01} \cosh (|\xi|)+\left(\xi_{v}^{12} X_{12}+\xi_{a}^{12} P_{12}\right) \frac{\sinh (|\xi|)}{|\xi|} \\
& P^{\prime 01}=P^{01} \cosh (|\xi|)+\left(\xi_{v}^{12} P_{12}-\xi_{a}^{12} X_{12}\right) \frac{\sinh (|\xi|)}{|\xi|} \\
& X^{\prime 12}=X^{12} \cosh (|\xi|)+\left(\xi_{v}^{12} X_{01}-\xi_{a}^{12} P_{01}\right) \frac{\sinh (|\xi|)}{|\xi|}
\end{aligned}
$$


$P^{\prime 12}=P^{12} \cosh (|\xi|)+\left(\xi_{v}^{12} P_{01}+\xi_{a}^{12} X_{01}\right) \frac{\sinh (|\xi|)}{|\xi|}$

$X_{i j}^{\prime}=X_{i j}, P_{i j}^{\prime}=P_{i j}$, for $i j \neq 12 ; \quad X^{\prime 0 i}=X^{0 i}, P^{\prime 0 i}=P^{0 i}$, for $i \neq 1$

where the absolute value $|\xi|$ for the areal-boost rapidity parameter is given by

$$
|\xi|^{2}=\left(\xi_{v}^{12}\right)^{2}+\left(\xi_{a}^{12}\right)^{2}
$$

The above areal rapidity boost parameters are defined in terms of the areal velocities and areal accelerations/forces of a new primed $C$-phase-space frame of reference which is moving with respect to the initial $C$-phase-space frame. Namely one has

$$
\tanh \left(\xi_{v}^{12}\right)=\frac{1}{c L_{P}} \frac{d X^{12}}{d t}, \quad \tanh \left(\xi_{a}^{12}\right)=\frac{1}{m_{P} c \mathcal{F}_{\max }} \frac{d P^{12}}{d t}
$$

When

$$
\xi_{v}^{12} \rightarrow \infty \Leftrightarrow \frac{d X^{12}}{d t} \rightarrow c L_{P} ; \quad \xi_{a}^{12} \rightarrow \infty \Leftrightarrow \frac{d P^{12}}{d t} \rightarrow m_{P} c \mathcal{F}_{\max }
$$

One can easily verify that the transformations (2.13) leave the interval invariant

$-\left(X^{\prime 0 i}\right)^{2}-\left(P^{\prime 0 i}\right)^{2}+\left(X^{\prime i j}\right)^{2}+\left(P^{\prime i j}\right)^{2}=-\left(X^{0 i}\right)^{2}-\left(P^{0 i}\right)^{2}+\left(X^{i j}\right)^{2}+\left(P^{i j}\right)^{2}$

and also leave invariant the bivector generalizations of the symplectic two-form

$$
-d X^{\prime 0 i} \wedge d P^{\prime 0 i}+d X^{\prime i j} \wedge d P^{\prime i j}=-d X^{0 i} \wedge d P^{0 i}+d X^{i j} \wedge d P^{i j}
$$

where

$$
\begin{gathered}
d X^{\prime 0 i} \wedge d P^{\prime 0 i}=-d P^{\prime 0 i} \wedge d X^{\prime 0 i} ; d X^{\prime i j} \wedge d P^{\prime i j}=-d P^{\prime i j} \wedge d X^{\prime i j} \\
d X^{\prime i j} \wedge d X^{\prime i j}=0 ; d P^{\prime i j} \wedge d P^{\prime i j}=0 ; \ldots \ldots .
\end{gathered}
$$

Naturally the transformations (2.13) solely affect the bivector components and do not affect the other polyvector components. The composition of two successive transformations of the form (2.13) yields a similar transformation (2.13) with the composition of rapidity parameters obeying similar relations as in eqs(1.6-1.9).

The transformations (2.13) can be generalized to tri-vectors and other higher grade polyvectors associated with the Clifford Phase Space. For example, in the trivector case we have the following transformations 


$$
\begin{gathered}
X^{\prime 012}=X^{012} \cosh (|\xi|)+\left(\xi_{v}^{123} X_{123}+\xi_{a}^{123} P_{123}\right) \frac{\sinh (|\xi|)}{|\xi|} \\
P^{\prime 012}=P^{012} \cosh (|\xi|)+\left(\xi_{v}^{123} P_{123}-\xi_{a}^{123} X_{123}\right) \frac{\sinh (|\xi|)}{|\xi|} \\
X^{\prime 123}=X^{123} \cosh (|\xi|)+\left(\xi_{v}^{123} X_{012}-\xi_{a}^{123} P_{012}\right) \frac{\sinh (|\xi|)}{|\xi|} \\
P^{\prime 123}=P^{123} \cosh (|\xi|)+\left(\xi_{v}^{123} P_{012}+\xi_{a}^{123} X_{012}\right) \frac{\sinh (|\xi|)}{|\xi|} \\
X_{i j k}^{\prime}=X_{i j k}, P_{i j k}^{\prime}=P_{i j k}, \quad \text { for } i j k \neq 123 \\
X^{\prime 0 i j}=X^{0 i j}, \quad P^{\prime 0 i j}=P^{0 i j}, \quad \text { for } i j \neq 12
\end{gathered}
$$

where the absolute value $|\xi|$ for the volume-boost rapidity parameter is given by

$$
|\xi|^{2}=\left(\xi_{v}^{123}\right)^{2}+\left(\xi_{a}^{123}\right)^{2}
$$

the volume rapidity boost parameters are defined in terms of the volume velocities and volume accelerations/forces of a new primed $C$-phase-space frame of reference which is moving with respect to the initial $C$-phase-space frame such that

$$
\tanh \left(\xi_{v}^{123}\right)=\frac{1}{c L_{P}^{2}} \frac{d X^{123}}{d t}, \quad \tanh \left(\xi_{a}^{123}\right)=\frac{1}{m_{P}^{2} c^{2} \mathcal{F}_{\max }} \frac{d P^{123}}{d t}
$$

The composition of two successive transformations (2.20) yields a similar transformation (2.20) with the composition of the rapidity parameters obeying similar relations as in eqs-(1.6-1.9).

One can easily verify that the transformations (2.20) leave the interval invariant

$\left(X^{\prime 0 i j}\right)^{2}+\left(P^{\prime 0 i j}\right)^{2}-\left(X^{\prime i j k}\right)^{2}-\left(P^{\prime i j k}\right)^{2}=\left(X^{0 i j}\right)^{2}+\left(P^{0 i j}\right)^{2}-\left(X^{i j k}\right)^{2}-\left(P^{i j k}\right)^{2}$

and also leave invariant the trivector generalizations of the symplectic two-form

$$
d X^{\prime 0 i j} \wedge d P^{\prime 0 i j}-d X^{\prime i j k} \wedge d P^{\prime i j k}=d X^{0 i j} \wedge d P^{0 i j}-d X^{i j k} \wedge d P^{i j k}
$$

Naturally the transformations (2.20) solely affect the trivector components and do not affect the other polyvector components. In this fashion one can construct 
the other transformations for the same grade polyvectors. One should notice that in $D=4$, for example, one has $X^{0123}, P^{0123}$ but there is no $X^{1234}, P^{1234}, \xi^{1234}$, then one must have in this $D=4$ case that $X^{\prime 0123}=X^{0123}, P^{\prime 0123}=P^{0123}$, implying that the pseudo-scalar components are invariant. The scalar components of the polyvectors are trivially invariant under $C$-(phase)-space coordinate transformations. The most general transformations in C-phase-space mix different grade components of the polyvectors $X^{M}, P^{M}$. These will be the subject of further investigation.

\subsection{A Clifford realization of velocity and acceleration/force boosts}

We begin by defining the phase space vector associated to a $D$-dim spacetime (in natural units $\hbar=G=c=b=1$ ) as

$$
\mathbf{Z}=x^{0} \gamma_{0}+p^{0} \beta_{0}+x^{1} \gamma_{1}+p^{1} \beta_{1}+\ldots \ldots
$$

In four spacetime dimensions, the signature of the $8 D$ Phase space $x^{0}, p^{0}, x^{1}, p^{1}, x^{2}, p^{2}, \ldots$ is chosen to be $(+,+,-,-,-,-\ldots-)$; i.e. there are two timelike and six spacelike directions. We choose to split the $8 D$ Clifford algebra generators into pairs of $\gamma^{\prime}$ s and $\beta$ 's as follows

$\Gamma_{0}=\gamma_{0} . \quad \Gamma_{1}=\gamma_{1}, \quad \Gamma_{2}=\gamma_{2} . \quad \Gamma_{3}=\gamma_{3} . \quad \Gamma_{4}=\beta_{0}, \quad \Gamma_{5}=\beta_{1}, \quad \Gamma_{6}=\beta_{2}, \quad \Gamma_{7}=\beta_{3}$

obeying the relations

$$
\left\{\gamma_{\mu}, \gamma_{\nu}\right\}=2 \eta^{\mu \nu} . \quad\left\{\beta_{\mu}, \beta_{\nu}\right\}=2 \eta^{\mu \nu} . \quad\left\{\gamma_{\mu}, \beta_{\nu}\right\}=0 . \quad\left\{\beta_{\mu}, \gamma_{\nu}\right\}=0
$$

Thus the squares of the basis generators are

$$
\gamma_{0}^{2}=\beta_{0}^{2}=1 . \quad \gamma_{1}^{2}=\beta_{1}^{2}=-1 . \quad \gamma_{2}^{2}=\beta_{2}^{2}=-1 . \quad \gamma_{3}^{2}=\beta_{3}^{2}=-1 . \quad \ldots .
$$

we also have the important relations

$$
\left(\gamma_{0} \gamma_{1}\right)\left(\gamma_{0} \gamma_{1}\right)=-\gamma_{0}^{2} \gamma_{1}^{2}=1 . \quad\left(\beta_{0} \beta_{1}\right)\left(\beta_{0} \beta_{1}\right)=-\beta_{0}^{2} \beta_{1}^{2}=1
$$

The real Clifford algebras $C l(2,6, R), C l(6,2, R)$ can be realized in terms of the matrix algebra $\mathcal{M}(8, \mathbf{H})$ given by $8 \times 8$ Quaternionic entries. Rather than working directly with a Clifford algebra in $8 D$ one may use instead the direct sum $C l(4) \oplus C l(4)$ with $16+16$ generators.

The identities

$\cosh (\|\xi\|)=\cosh ^{2}\left(\frac{1}{2}\|\xi\|\right)+\sinh ^{2}\left(\frac{1}{2}\|\xi\|\right), \quad \cosh ^{2}\left(\frac{1}{2}\|\xi\|\right)-\sinh ^{2}\left(\frac{1}{2}\|\xi\|\right)=1$ 


$$
\sinh (\|\xi\|)=2 \sinh \left(\frac{1}{2}\|\xi\|\right) \cosh \left(\frac{1}{2}\|\xi\|\right) .
$$

must be used always in order to reproduce the $\cosh (\|\xi\|), \sinh (\|\xi\|)$ from their half-values. For simplicity and without loss of generality we shall focus on the transformations corresponding to a four-dim phase space associated with a twodim spacetime. There are four relevant bivectors

$$
\gamma_{0} \wedge \gamma_{1}, \beta_{0} \wedge \beta_{1} . \quad \gamma_{0} \wedge \beta_{1}, \beta_{0} \wedge \gamma_{1}
$$

the first two bivectors in (2.31) correspond to ordinary velocity boosts/rotations in the $x_{0}-x_{1}$ and $p_{0}-p_{1}$ planes, respectively, whereas the last two bivectors are acceleration/force boosts mixing the $T$ time coordinate with the momentum $P_{1}$, and the energy $E$ with the coordinate $X_{1}$, respectively. For instance, after setting $c=b=1$, the transformation

$$
\begin{gathered}
\left(\cosh \left(\frac{1}{2} \xi_{a}\right)+\gamma_{0} \beta_{1} \sinh \left(\frac{1}{2} \xi_{a}\right)\right)\left(T \gamma_{0}+E \beta_{0}+X \gamma_{1}+P \beta_{1}\right)\left(\cosh \left(\frac{1}{2} \xi_{a}\right)-\gamma_{0} \beta_{1} \sinh \left(\frac{1}{2} \xi_{a}\right)\right)= \\
T^{\prime} \gamma_{0}+E^{\prime} \beta_{0}+X^{\prime} \gamma_{1}+P^{\prime} \beta_{1}
\end{gathered}
$$

yields

$$
T^{\prime}=T \cosh \left(\xi_{a}\right)-P \sinh \left(\xi_{a}\right), \quad P^{\prime}=P \cosh \left(\xi_{a}\right)-T \sinh \left(\xi_{a}\right)
$$

and

$$
E^{\prime}=E, \quad X^{\prime}=X
$$

such that the interval is invariant

$$
\left(T^{\prime}\right)^{2}+\left(E^{\prime}\right)^{2}-\left(X^{\prime}\right)^{2}-\left(P^{\prime}\right)^{2}=(T)^{2}+(E)^{2}-(X)^{2}-(P)^{2}
$$

Whereas the transformation

$$
\begin{gathered}
\left(\cosh \left(\frac{1}{2} \xi_{a}\right)+\beta_{0} \gamma_{1} \sinh \left(\frac{1}{2} \xi_{a}\right)\right)\left(T \gamma_{0}+E \beta_{0}+X \gamma_{1}+P \beta_{1}\right)\left(\cosh \left(\frac{1}{2} \xi_{a}\right)-\beta_{0} \gamma_{1} \sinh \left(\frac{1}{2} \xi_{a}\right)\right)= \\
T^{\prime} \gamma_{0}+E^{\prime} \beta_{0}+X^{\prime} \gamma_{1}+P^{\prime} \beta_{1}
\end{gathered}
$$

yields

$$
E^{\prime}=E \cosh \left(\xi_{a}\right)-X \sinh \left(\xi_{a}\right), \quad X^{\prime}=X \cosh \left(\xi_{a}\right)-E \sinh \left(\xi_{a}\right)
$$

and

$$
P^{\prime}=P, \quad T^{\prime}=T
$$

such that the interval (2.34) is invariant.

The transformations involving the rotor

$$
\mathbf{R}=\cosh \left(\frac{1}{2} \xi_{v}\right)+\gamma_{0} \gamma_{1} \sinh \left(\frac{1}{2} \xi_{v}\right), \quad \mathbf{R}^{-1}=\cosh \left(\frac{1}{2} \xi_{v}\right)-\gamma_{0} \gamma_{1} \sinh \left(\frac{1}{2} \xi_{v}\right)
$$


lead to the ordinary velocity Lorentz boosts of the $X, T$ coordinates and leave invariant the energy-momentum $E, P$.

The transformations involving the rotor

$$
\mathbf{R}=\cosh \left(\frac{1}{2} \xi_{v}\right)+\beta_{0} \beta_{1} \sinh \left(\frac{1}{2} \xi_{v}\right), \mathbf{R}^{-1}=\cosh \left(\frac{1}{2} \xi_{v}\right)-\beta_{0} \beta_{1} \sinh \left(\frac{1}{2} \xi_{v}\right)
$$

lead to the ordinary velocity Lorentz boosts of the $E, P$ energy-momentum and leave invariant the $X, T$ coordinates.

Finally, given $\mathbf{Z}=T \gamma_{0}+E \beta_{0}+X \gamma_{1}+P \beta_{1}$ the transformation we are looking for which combines both velocity and acceleration/force boosts simultaneously is

$$
\mathbf{Z}^{\prime}=\mathbf{R}_{1} \mathbf{R}_{2} \mathbf{Z} \mathbf{R}_{2}^{-1} \mathbf{R}_{1}^{-1}
$$

the first rotor is given by

$$
\begin{gathered}
\mathbf{R}_{1}=\cosh (\|\alpha\|)-\left[\alpha_{v} \gamma_{0} \gamma_{1}+\alpha_{a} \gamma_{1} \beta_{0}\right] \frac{\sinh (\|\alpha\|)}{\|\alpha\|} . \\
\tilde{\mathbf{R}}_{1}=\mathbf{R}_{1}^{-1}=\cosh (\|\alpha\|)+\left[\alpha_{v} \gamma_{0} \gamma_{1}+\alpha_{a} \gamma_{1} \beta_{0}\right] \frac{\sinh (\|\alpha\|)}{\|\alpha\|}
\end{gathered}
$$

and where $\|\alpha\| \equiv \sqrt{\left(\alpha_{v}\right)^{2}+\left(\alpha_{a}\right)^{2}}$. The other rotor is

$$
\begin{gathered}
\mathbf{R}_{2}=\cosh (\|\alpha\|)-\left[\alpha_{v} \beta_{0} \beta_{1}+\alpha_{a} \gamma_{0} \beta_{1}\right] \frac{\sinh (\|\alpha\|)}{\|\alpha\|} . \\
\tilde{\mathbf{R}}_{2}=\mathbf{R}_{2}^{-1}=\cosh (\|\alpha\|)+\left[\alpha_{v} \beta_{0} \beta_{1}+\alpha_{a} \gamma_{0} \beta_{1}\right] \frac{\sinh (\|\alpha\|)}{\left\|\alpha^{\prime}\right\|} .
\end{gathered}
$$

Therefore, the transformation

$$
\begin{gathered}
\mathbf{R}_{1} \mathbf{R}_{2}\left(T \gamma_{0}+E \beta_{0}+X \gamma_{1}+P \beta_{1}\right) \mathbf{R}_{2}^{-1} \mathbf{R}_{1}^{-1}= \\
T^{\prime} \gamma_{0}+E^{\prime} \beta_{0}+X^{\prime} \gamma_{1}+P^{\prime} \beta_{1}
\end{gathered}
$$

with

$$
\begin{gathered}
\mathbf{R}_{1} \mathbf{R}_{2}=\cosh ^{2}(\|\alpha\|)+\gamma_{0} \gamma_{1} \beta_{0} \beta_{1} \sinh ^{2}(\|\alpha\|)- \\
\left(\alpha_{v} \gamma_{0} \gamma_{1}+\alpha_{v} \beta_{0} \beta_{1}+\alpha_{a} \beta_{0} \gamma_{1}+\alpha_{a} \gamma_{0} \beta_{1}\right) \frac{\sinh (\|\alpha\|) \cosh (\|\alpha\|)}{\alpha} \\
\left(\mathbf{R}_{1} \mathbf{R}_{2}\right)^{-1}=\mathbf{R}_{2}^{-1} \mathbf{R}_{1}^{-1}=\cosh ^{2}(\|\alpha\|)+\gamma_{0} \gamma_{1} \beta_{0} \beta_{1} \sinh ^{2}(\|\alpha\|)+ \\
\left(\alpha_{v} \gamma_{0} \gamma_{1}+\alpha_{v} \beta_{0} \beta_{1}+\alpha_{a} \beta_{0} \gamma_{1}+\alpha_{a} \gamma_{0} \beta_{1}\right) \frac{\sinh (\|\alpha\|) \cosh (\|\alpha\|)}{\alpha}
\end{gathered}
$$


involving the rotors $(2.40,2.41)$ associated with the generalized rapidity parameters $\|\alpha\| \equiv \sqrt{\left(\alpha_{v}\right)^{2}+\left(\alpha_{a}\right)^{2}}$ lead to the sought-after velocity and acceleration/force boosts transformations mixing the coordinates and momenta and which leave invariant the interval

$$
\left(T^{\prime}\right)^{2}+\left(E^{\prime}\right)^{2}-\left(X^{\prime}\right)^{2}-\left(P^{\prime}\right)^{2}=(T)^{2}+(E)^{2}-(X)^{2}-(P)^{2}
$$

However, these transformations instead of being displayed explicitly in terms of the generalized rapidity parameter $\|\xi\|=\sqrt{\left(\xi_{v}\right)^{2}+\left(\xi_{a}\right)^{2}}$, as indicated by eqs- $(1.2,1.4)$, we have the transformations (2.42) written explicitly in terms of the parameters $\alpha_{v}, \alpha_{a},\|\alpha\|$ and the Clifford algebra generators. One can recast the latter parameters in terms of $\xi_{v}, \xi_{a},\|\xi\|$ after equating the set of variables $T^{\prime}, E^{\prime}, X^{\prime}, P^{\prime}$ in eq-(2.42) with those appearing in eqs-(1.2).

For example, by focusing on the factors of the $T$ variable appearing in both eqs-(2.42) and eqs-(1.2), after lengthy algebra and recurring to the results

$$
\begin{gathered}
\left(\gamma_{0} \gamma_{1} \beta_{0} \beta_{1}\right) \gamma_{0}\left(\gamma_{0} \gamma_{1} \beta_{0} \beta_{1}\right)=-\gamma_{0} \\
\left(\alpha_{v} \gamma_{0} \gamma_{1}+\alpha_{v} \beta_{0} \beta_{1}+\alpha_{a} \beta_{0} \gamma_{1}+\alpha_{a} \gamma_{0} \beta_{1}\right) \gamma_{0}\left(\alpha_{v} \gamma_{0} \gamma_{1}+\alpha_{v} \beta_{0} \beta_{1}+\alpha_{a} \beta_{0} \gamma_{1}+\alpha_{a} \gamma_{0} \beta_{1}\right)=0
\end{gathered}
$$

and the identities for the hyperbolic functions, one arrives at

$$
\begin{gathered}
\cosh ^{4} \alpha-\sinh ^{4} \alpha=\left(\cosh ^{2} \alpha+\sinh ^{2} \alpha\right)\left(\cosh ^{2} \alpha-\sinh ^{2} \alpha\right)=\cosh (2 \alpha)=\cosh (\xi) \\
\left(\cosh ^{2} \alpha-\sinh ^{2} \alpha\right) \frac{\sinh (\alpha) \cosh (\alpha)}{\alpha}=\frac{\sinh (2 \alpha)}{2 \alpha}=\frac{\sinh (\xi)}{\xi}
\end{gathered}
$$

from which one obtains the desired relationship among the rapidity parameters

$$
\xi_{v}=2 \alpha_{v}, \quad \xi_{a}=2 \alpha_{a}, \quad \xi=\sqrt{\left(\xi_{v}\right)^{2}+\left(\xi_{a}\right)^{2}}=2 \alpha=2 \sqrt{\left(\alpha_{v}\right)^{2}+\left(\alpha_{a}\right)^{2}}
$$

The reason one has to recur to the transformations of the form $(2.42)$ is because

$$
\begin{gathered}
\left(\alpha_{v} \gamma_{0} \gamma_{1}+\alpha_{v} \beta_{0} \beta_{1}+\alpha_{a} \beta_{0} \gamma_{1}+\alpha_{a} \gamma_{0} \beta_{1}\right)^{2}= \\
2 \alpha^{2}\left(1+\gamma_{0} \gamma_{1} \beta_{0} \beta_{1}\right) \neq 2 \alpha^{2}
\end{gathered}
$$

eq- $(2.50 \mathrm{a})$ is a result of

$$
\left\{\gamma_{0} \gamma_{1}+\beta_{0} \beta_{1}, \gamma_{1} \beta_{0}+\gamma_{0} \beta_{1}\right\}=0
$$

One can explicitly verify that after using the rest of the relations

$$
\left(\gamma_{0} \gamma_{1} \beta_{0} \beta_{1}\right) \gamma_{1}\left(\gamma_{0} \gamma_{1} \beta_{0} \beta_{1}\right)=-\gamma_{1}
$$




$$
\begin{aligned}
& \left(\gamma_{0} \gamma_{1} \beta_{0} \beta_{1}\right) \beta_{0}\left(\gamma_{0} \gamma_{1} \beta_{0} \beta_{1}\right)=-\beta_{0} \\
& \left(\gamma_{0} \gamma_{1} \beta_{0} \beta_{1}\right) \beta_{1}\left(\gamma_{0} \gamma_{1} \beta_{0} \beta_{1}\right)=-\beta_{1}
\end{aligned}
$$

$\left(\alpha_{v} \gamma_{0} \gamma_{1}+\alpha_{v} \beta_{0} \beta_{1}+\alpha_{a} \beta_{0} \gamma_{1}+\alpha_{a} \gamma_{0} \beta_{1}\right) \gamma_{1}\left(\alpha_{v} \gamma_{0} \gamma_{1}+\alpha_{v} \beta_{0} \beta_{1}+\alpha_{a} \beta_{0} \gamma_{1}+\alpha_{a} \gamma_{0} \beta_{1}\right)=0$

$\left(\alpha_{v} \gamma_{0} \gamma_{1}+\alpha_{v} \beta_{0} \beta_{1}+\alpha_{a} \beta_{0} \gamma_{1}+\alpha_{a} \gamma_{0} \beta_{1}\right) \beta_{0}\left(\alpha_{v} \gamma_{0} \gamma_{1}+\alpha_{v} \beta_{0} \beta_{1}+\alpha_{a} \beta_{0} \gamma_{1}+\alpha_{a} \gamma_{0} \beta_{1}\right)=0$

$\left(\alpha_{v} \gamma_{0} \gamma_{1}+\alpha_{v} \beta_{0} \beta_{1}+\alpha_{a} \beta_{0} \gamma_{1}+\alpha_{a} \gamma_{0} \beta_{1}\right) \beta_{1}\left(\alpha_{v} \gamma_{0} \gamma_{1}+\alpha_{v} \beta_{0} \beta_{1}+\alpha_{a} \beta_{0} \gamma_{1}+\alpha_{a} \gamma_{0} \beta_{1}\right)=0$

and eqs-(2.45-2.48), it leads to the full set of transformations given by eqs-(1.2).

In general, in higher spacetime dimensions than $D=2$ (higher dimensional phase spaces greater than 4), one can have velocity and acceleration boosts along arbitrary spatial directions $X^{i}$ with $i=1,2,3, \ldots ., D-1$. Due to the fact that

$$
\begin{gathered}
{\left[\alpha_{v}^{i} \gamma_{0} \gamma_{i}+\alpha_{a}^{i} \gamma_{i} \beta_{0}\right]\left[\alpha_{v}^{j} \gamma_{0} \gamma_{j}+\alpha_{a}^{j} \gamma_{j} \beta_{0}\right]=} \\
\left(\alpha_{v}^{i}\right)^{2}+\left(\alpha_{a}^{i}\right)^{2}+\alpha_{v}^{[i} \alpha_{a}^{j]} \gamma_{0} \gamma_{i j} \beta_{0} \neq\left(\alpha_{v}^{i}\right)^{2}+\left(\alpha_{a}^{i}\right)^{2}=\alpha^{2}
\end{gathered}
$$

the rotor given by

$$
\mathbf{R}_{1}=\cosh (\|\alpha\|)-\left[\alpha_{v}^{i} \gamma_{0} \gamma_{i}+\alpha_{a}^{i} \gamma_{i} \beta_{0}\right] \frac{\sinh (\|\alpha\|)}{\|\alpha\|} .
$$

does no longer satisfy the condition $\tilde{\mathbf{R}}_{1}=\mathbf{R}_{1}^{-1}$, so now one has that

$$
\tilde{\mathbf{R}}_{1}=\cosh (\|\alpha\|)+\left[\alpha_{v}^{i} \gamma_{0} \gamma_{i}+\alpha_{a}^{i} \gamma_{i} \beta_{0}\right] \frac{\sinh (\|\alpha\|)}{\|\alpha\|} \neq \mathbf{R}_{1}^{-1}
$$

where now one must sum over all the spatial directions : $\|\alpha\| \equiv \sqrt{\left(\alpha_{v}^{i}\right)^{2}+\left(\alpha_{a}^{i}\right)^{2}}$. The other rotor

$$
\mathbf{R}_{2}=\cosh (\|\alpha\|)-\left[\alpha_{v}^{i} \beta_{0} \beta_{i}+\alpha_{a}^{i} \gamma_{0} \beta_{i}\right] \frac{\sinh (\|\alpha\|)}{\|\alpha\|} .
$$

does no longer satisfy the condition $\tilde{\mathbf{R}}_{2}=\mathbf{R}_{2}^{-1}$, so now one has that

$$
\tilde{\mathbf{R}}_{2}=\cosh (\|\alpha\|)+\left[\alpha_{v}^{i} \beta_{0} \beta_{i}+\alpha_{a}^{i} \gamma_{0} \beta_{i}\right] \frac{\sinh (\|\alpha\|)}{\|\alpha\|} \neq \mathbf{R}_{2}^{-1}
$$

In order to obtain the most general transformations [10], in units $\hbar=G=c=$ $b=1$,

$$
\begin{aligned}
& T^{\prime}=T \cosh \xi+\left(\xi_{v}^{i} X_{i}+\xi_{a}^{i} P_{i}\right) \frac{\sinh \xi}{\xi} \\
& E^{\prime}=E \cosh \xi+\left(\xi_{v}^{i} P_{i}-\xi_{a}^{i} X_{i}\right) \frac{\sinh \xi}{\xi}
\end{aligned}
$$




$$
\begin{aligned}
& X^{\prime i}=X^{i}+X_{j}\left(\xi_{v}^{i} \xi_{v}^{j}+\xi_{a}^{i} \xi_{a}^{j}\right) \frac{\cosh \xi-1}{\xi^{2}}+\left(\xi_{v}^{i} T-\xi_{a}^{i} E\right) \frac{\sinh \xi}{\xi} . \\
& P^{\prime i}=P^{i}+P_{j}\left(\xi_{v}^{i} \xi_{v}^{j}+\xi_{a}^{i} \xi_{a}^{j}\right) \frac{\cosh \xi-1}{\xi^{2}}+\left(\xi_{v}^{i} E+\xi_{a}^{i} T\right) \frac{\sinh \xi}{\xi} .
\end{aligned}
$$

where the effective boost parameter $\xi$ is defined in terms of the velocity and acceleration boosts parameters $\xi_{v}^{i}, \xi_{a}^{i}$, respectively, as

$$
\xi \equiv \sqrt{\left(\xi_{v}^{i}\right)^{2}+\left(\xi_{a}^{i}\right)^{2}}, \quad i=1,2,3, \ldots \ldots, D-1
$$

one would need to find a more complicated expression for the rotors. In particular, one may propose a transformation of the form

$$
\begin{gathered}
\left(\mathbf{R}_{1}^{(1)} \mathbf{R}_{2}^{(1)} \ldots \ldots . . \mathbf{R}_{1}^{(D-1)} \mathbf{R}_{2}^{(D-1)}\right) \mathbf{Z}\left(\left(\mathbf{R}_{2}^{(D-1)}\right)^{-1}\left(\mathbf{R}_{1}^{(D-1)}\right)^{-1} \ldots \ldots\left(\mathbf{R}_{2}^{(1)}\right)^{-1}\left(\mathbf{R}_{1}^{(1)}\right)^{-1}\right)= \\
T^{\prime} \gamma_{0}+E^{\prime} \beta_{0}+X^{\prime i} \gamma_{i}+P^{\prime i} \beta_{i} ; i=1,2,3, \ldots \ldots, D-1
\end{gathered}
$$

which leaves invariant the interval $(T)^{2}+(E)^{2}-\left(X^{i}\right)^{2}-\left(P^{i}\right)^{2}$, to see whether or not it reproduces the transformations (2.55) after one finds the relationship among the rapidity parameters $\xi, \xi_{v}^{i}, \xi_{a}^{i}$ and $\alpha, \alpha_{v}^{i}, \alpha_{a}^{i}$. Each pair of rotors $\mathbf{R}_{1}^{(i)} \mathbf{R}_{2}^{(i)}$ for $i=1,2,3, \ldots, D-1$ has the same functional form as those provided by eqs- $(2.40,2.41)$ with the provision that one uses one pair of rapidity parameters $\alpha_{v}^{i}, \alpha_{a}^{i}$ for each different value of $i=1,2,3, \ldots, D-1$. To verify this is beyond the scope of this work due to cumbersome algebra and will be the study of further investigations.

Another procedure is to write the $U(p, q)$ algebra generators in terms of the $S O(2 p, 2 q)$ algebra generators and, which in turn, can be written in terms of the bivectors generators of the real $C l(2 p, 2 q)$ algebra. It is well known to the experts that the $U(N)$ generators $E_{i j}$ can be written as bilinears of oscillators, which in turn, are given by linear combinations of the $S O(2 N)$ generators. When the velocity and acceleration/force boosts point along one of the spatial axis directions, like $X^{1}$, all values of $\xi_{v}^{i}, \xi_{a}^{i}$ are zero except when $i=1$, and one then recovers the transformations (1.2) from eqs-(2.55) and (2.57). The coordinates and momenta $X^{i}, P^{i}$ remain invariant when $i \neq 1$.

One can generalize the construction of this subsection to the case of areal velocity and areal acceleration/force boosts, etc..... The most general transformations of the form $\mathbf{Z}^{\prime}=\mathbf{R Z R} \mathbf{R}^{-1}$ and involving a rotor given by eqs-(2.5, 2.6) will mix the different grade components. We avoided the use of complex numbers (complex parameters). Nevertheless one could have worked with complexified Clifford algebras. Symplectic Clifford algebras [16] should also play an important role in Clifford Phase Spaces. The latter algebras are crucial in the formulation of Clifford super-analysis and the construction of superspaces with bosonic and Grassmanian valued coordinates. 


\section{A Clifford Phase Space Gravitational Theory}

A brief presentation of the formulation of $C$-space gravity from a Clifford bundle structure perspective is needed before one can construct a Clifford Phase Space Gravitational Theory. A review of the formulation of gravity and spin from a local conformal-affine symmetry point of view can be found in [20]. A geometric approach to $f(R)$ gravity with torsion within the Jet-bundle framework can be found in [21]. In particular, the torsion and curvature tensors can be chosen as fiber Jet-bundle coordinates which permit a simpler study of the symmetries and conservation laws of the theory.

Let us begin with a Clifford gauge field theory based on the $C l(4,1 ; R)$ algebra acting on the fibers associated with a Clifford fibre bundle constructed over a Clifford base space manifold associated to a $C l(3,1 ; R)$ algebra, and whose polyvector valued coordinates are $\mathbf{X}=X^{M} \gamma_{M}$. The $C l(4,1 ; R)$-valued gauge field $A_{M}$ is given by

$A_{M}=A_{M}^{I} \gamma_{I}=A_{M}^{0} \mathbf{1}+A_{M}^{i} \gamma_{i}+A_{M}^{i_{1} i_{2}} \gamma_{i_{1} i_{2}}+\ldots \ldots \ldots+A_{M}^{i_{1} i_{2} \ldots . . i_{5}} \gamma_{i_{1} i_{2} \ldots i_{5}}$

One can decompose the $C l(4,1)$ poly-vector valued indices $I$ as

$$
\begin{gathered}
A_{M}^{i}=\left(A_{M}^{a}, A_{M}^{5}\right) ; \quad A_{M}^{i_{1} i_{2}}=\left(A_{M}^{a_{1} a_{2}}, A_{M}^{a 5}\right), \ldots \\
A_{M}^{i_{1} i_{2} i_{3}}=\left(A_{M}^{a_{1} a_{2} a_{3}}, A_{M}^{a_{1} a_{2} 5}\right), \ldots \ldots, A_{M}^{i_{1} i_{2} i_{3} i_{4} i_{5}}=A_{M}^{a_{1} a_{2} a_{3} a_{4} 5}
\end{gathered}
$$

the indices $a_{1}, a_{2}, \ldots$ span the values $1,2,3,4$, whereas the indices $i_{1}, i_{2}, \ldots$ span the values $1,2, \ldots, 4,5$. In this way one extract the generalized beins $E_{M}^{A}$ from eq-(3.2) as follows

$E_{M}^{0} \leftrightarrow A_{M}^{5}, \quad E_{M}^{a} \leftrightarrow A_{M}^{a 5}, \quad E_{M}^{a_{1} a_{2}} \leftrightarrow A_{M}^{a_{1} a_{2} 5}, \ldots \ldots, E_{M}^{a_{1} a_{2} \ldots a_{4}} \leftrightarrow A_{M}^{a_{1} a_{2} a_{3} a_{4} 5}$

after a suitable scaling by powers of a fundamental length (Planck scale). Similarly, the (noncommuting) polyvector valued momentum (translation operators) in the tangent $C$-space are identified as

$$
P_{0} \leftrightarrow \gamma_{5}, P_{a} \leftrightarrow \gamma_{a 5}, P_{a_{1} a_{2}} \leftrightarrow \gamma_{a_{1} a_{2} 5}, \ldots ., P_{a_{1} a_{2} \ldots a_{4}} \leftrightarrow \gamma_{a_{1} a_{2} a_{3} a_{4} 5}
$$

The ordinary spin connection $\omega_{\mu}^{a_{1} a_{2}}$ of spacetime sits inside the components

$$
\Omega_{M}^{a_{1} a_{2}}=A_{M}^{a_{1} a_{2}}=\left\{A_{0}^{a_{1} a_{2}}, A_{\mu}^{a_{1} a_{2}}, A_{\mu_{1} \mu_{2}}^{a_{1} a_{2}}, \ldots ., A_{\mu_{1} \mu_{2} \mu_{3} \mu_{4}}^{a_{1} a_{2}}\right\}
$$

in particular, by identifying $A_{\mu}^{a_{1} a_{2}}=\omega_{\mu}^{a_{1} a_{2}}$. The generalized poly-rotations generators $J_{A}$ correspond to those basis generators which do not contain the 5-th direction

$J_{a} \leftrightarrow \gamma_{a}, J_{a_{1} a_{2}} \leftrightarrow \gamma_{a_{1} a_{2}}, J_{a_{1} a_{2} a_{3}} \leftrightarrow \gamma_{a_{1} a_{2} a_{3}}, \ldots . ., J_{a_{1} a_{2} \ldots . a_{4}} \leftrightarrow \gamma_{a_{1} a_{2} a_{3} a_{4}}$ 
one must also include the unit generator $\gamma_{0}=J_{0}=\mathbf{1}$ as well.

Therefore, after recurring to eqs-(3.1-3.6) the $C l(4,1)$-valued gauge field $A_{M}$ in eq-(3.1) can be rewritten in terms of generalized poly-rotations $J_{A}$ and polytranslations $P_{A}$ (in the tangent $C$-space) as

$$
A_{M}=A_{M}^{I} \gamma_{I}=\Omega_{M}^{A} J_{A}+E_{M}^{A} P_{A}
$$

This is the Clifford space analog of gauging the de Sitter group $S O(4,1)$ so that the "translations" along the four-dimensional directions $P_{a}$ can be viewed as the rotations involving the internal 5 -th direction $J_{a 5}$. The Poincare group in four dimensions $S O(3,1) \times{ }_{s} T_{4}$ (semi-direct product of Lorentz with translations) can be obtained as a group contraction of the de Sitter group $S O(4,1)$ by taking the throat size of the four-dim hyperboloid (embedded in five flat dimensions) to infinity.

Therefore, the field strength associated with the $C l(4,1)$ valued gauge field $A_{M}=A_{M}^{I} \gamma_{I}$ can be decomposed into a rotational and translational piece as follows

$$
\begin{gathered}
F_{M N}^{K} \gamma_{K}=\left(\partial_{M} A_{N}^{K}-\partial_{N} A_{M}^{K}\right) \gamma_{K}+\left[A_{M}^{I} \gamma_{I}, A_{N}^{J} \gamma_{J}\right]= \\
\mathcal{R}_{M N}^{A} J_{A}+\mathcal{T}_{M N}^{A} P_{A}
\end{gathered}
$$

after recurring to the commutators

$$
\left[J_{A}, J_{B}\right]=f_{A B C} J_{C},\left[J_{A}, P_{B}\right]=f_{A B C}^{\prime} P_{C},\left[P_{A}, P_{B}\right]=f_{A B C}^{\prime \prime} J_{C}
$$

the above commutators are the $C l(4,1)$ algebraic extension of the $S O(4,1)$ commutators. We have set the length scale to unity in the last term of (3.14). Such scale must be included in order to match units. In [22] the whole construction was generalized to include the conformal group $S O(4,2)$ as well.

One can convert the gauge group indices of the field strengths into base space indices by contracting indices with the (inverse) beins as follows $\mathcal{R}_{M N}^{A} E_{A}^{L}=$ $\mathcal{R}_{M N}^{L}$. In the diagonal gauge, when the mixed grade components of the (inverse) beins $E_{L}^{A}, E_{A}^{L}$ are zero, one has as an example for the conversion process of gauge group indices of into base space indices the following

$$
F_{M N}^{a_{1} a_{2}} \leftrightarrow \mathcal{R}_{M N}^{a_{1} a_{2}} \Rightarrow \mathcal{R}_{M N}^{a_{1} a_{2}} E_{a_{1} a_{2}}^{\rho_{1} \rho_{2}}=\mathcal{R}_{M N}^{\left[\rho_{1} \rho_{2}\right]}
$$

such that the de Siter $(\mathrm{dS})$ curvature tensor $R_{[\mu \nu]}^{(d S)\left[\rho_{1} \rho_{2}\right]}$ sits now inside the components

$$
\mathcal{R}_{[M N]}^{\left[\rho_{1} \rho_{2}\right]}=\left\{\mathcal{R}_{[\mu \nu]}^{\left[\rho_{1} \rho_{2}\right]}, \mathcal{R}_{\left[\mu_{1} \mu_{2} \nu_{1} \nu_{2}\right]}^{\left[\rho_{1} \rho_{2}\right]}, \mathcal{R}_{\left[\mu_{1} \mu_{2} \mu_{3} \nu_{1} \nu_{2} \nu_{3}\right]}^{\left[\rho_{1} \rho_{2}\right]}, \ldots . .\right\}
$$

In particular one can verify that one recovers the de Sitter (dS) curvature tensor $R_{[\mu \nu]}^{(d S)\left[\rho_{1} \rho_{2}\right]}$ as a sum of the Riemannian curvature tensor plus corrections involving the vielbeins multiplying the cosmological constant. In differential form notation one has that the de Sitter curvature 2-form is $R^{(d S)}=R(\omega)+l^{-2} e \wedge e$. 
The MacDowell-Mansouri-Chamsedinne-West action in four-dimensions is given by $\int \mathcal{R} \wedge \mathcal{R}$, which in turn, becomes the Gauss-Bonnet topological invariant term $\int R \wedge R$; the Einstein-Hilbert action $l^{-2} \int R \wedge e \wedge e$, and the cosmological constant term $l^{-4} \int e \wedge e \wedge e \wedge e$.

After setting the length scale $l=1$ one has that the components of the modified curvature 2 -form due to the Clifford algebraic structure are given by

$$
\begin{gathered}
\mathcal{R}_{\mu \nu}^{a_{1} a_{2}}=\partial_{[\mu} \Omega_{\nu]}^{a_{1} a_{2}}+\Omega_{\mu}^{m 5} \Omega_{\nu}^{r 5}<\left[\gamma_{m 5}, \gamma_{r 5}\right] \gamma^{a_{1} a_{2}}>+\Omega_{\mu}^{m n} \Omega_{\nu}^{r s}<\left[\gamma_{m n}, \gamma_{r s}\right] \gamma^{a_{1} a_{2}}>+ \\
\Omega_{\mu}^{m n p} \Omega_{\nu}^{r s t}<\left[\gamma_{m n p}, \gamma_{r s t}\right] \gamma^{a_{1} a_{2}}>+\Omega_{\mu}^{m n p q} \Omega_{\nu}^{r s t u}<\left[\gamma_{m n p q}, \gamma_{r s t u}\right] \gamma^{a_{1} a_{2}}>+ \\
\Omega_{\mu}^{m n p q k} \Omega_{\nu}^{r s t u v}<\left[\gamma_{m n p q k}, \gamma_{r s t u v}\right] \gamma^{a_{1} a_{2}}>
\end{gathered}
$$

where the brackets $<\left[\gamma_{m n}, \gamma_{r}\right] \gamma^{a}>,<\left[\gamma_{m n p q}, \gamma_{r s t}\right] \gamma^{a}>$ indicate the scalar part of the product of the $C l(4,1)$ algebra elements; i.e it extracts the $C l(4,1)$ invariant contribution. For example,

$<\left[\gamma_{m n}, \gamma_{r}\right] \gamma^{a}>=<-\eta_{m r} \gamma_{n} \gamma^{a}>+<\eta_{n r} \gamma_{m} \gamma^{a}>=-\eta_{m r} \delta_{n}^{a}+\eta_{n r} \delta_{m}^{a}$

Therefore one can verify that de Sitter curvature tensor is given by the first line of (3.12). The modified torsion 2-form due to the Clifford algebraic structure is given by

$$
\begin{aligned}
\mathcal{T}_{\mu \nu}^{a} & =\mathcal{R}_{\mu \nu}^{a 5}=\partial_{[\mu} \Omega_{\nu]}^{a 5}+\Omega_{\mu}^{m n} \Omega_{\nu}^{r 5}<\left[\gamma_{m n}, \gamma_{r 5}\right] \gamma^{a 5}>+ \\
\Omega_{\mu}^{m} \Omega_{\nu}^{r} & <\left[\gamma_{m}, \gamma_{r}\right] \gamma^{a 5}>+\Omega_{\mu}^{m n p} \Omega_{\nu}^{r s t}<\left[\gamma_{m n p}, \gamma_{r s t}\right] \gamma^{a 5}>+ \\
\Omega_{\mu}^{m n p q} \Omega_{\nu}^{r s t u} & <\left[\gamma_{m n p q}, \gamma_{r s t u}\right] \gamma^{a 5}>+\Omega_{\mu}^{m n p q k} \Omega_{\nu}^{r s t u v}<\left[\gamma_{m n p q k}, \gamma_{r s t u v}\right] \gamma^{a 5}>.
\end{aligned}
$$

Form (3.14) one can see that the $C l(4,1)$-algebraic expression for the torsion $\mathcal{T}_{\mu \nu}^{a}$ contains the standard expression for the torsion in Riemann-Cartan spacetimes. The Riemann-Cartan torsion is given by the first line of (3.14).

To finalize this discussion we recall [22] where one can define the doubleindex generators

$$
J_{A B}=\left[\gamma_{A}, \gamma_{B}\right]=f_{A B}^{C} \gamma_{C} \Rightarrow \Omega_{M}^{A B} J_{A B}=\Omega_{M}^{A B} f_{A B}^{C} \gamma_{C}=\Omega_{M}^{C} \gamma_{C}
$$

from which one learns that $\Omega_{M}^{A B} f_{A B}^{C}=\Omega_{M}^{C}$ so the rotational part of the curvature can be rewritten in double-index notation as

$$
\mathcal{R}_{M N}^{C} \gamma_{C}=\mathcal{R}_{M N}^{C} f_{C}^{A B} J_{A B}=\mathcal{R}_{M N}^{A B} J_{A B}
$$

and will allow us to convert group indices into base space indices

$$
\mathcal{R}_{M N}^{A B} E_{A}^{P} E_{B}^{Q}=\mathcal{R}_{M N}^{P Q} \Rightarrow \mathcal{R}_{M N}{ }_{M Q}^{M Q}=\mathcal{R}_{N}^{Q} \Rightarrow \mathcal{R}_{N}^{N}=\mathcal{R}
$$


hence, eq-(3.17) will enable us to construct the $C$-space scalar curvature $\mathcal{R}$ and build the analog of the Einstein-Hilbert action. For a detailed discussion of a Clifford gauge theory approach to gravity with torsion see [22].

The procedure that led to the construction of a Born reciprocal general relativity theory in a curved $4 D$ spacetime [17] (based in gauging the Quaplectic group) can be extended to a theory involving polyvector-valued coordinates and momenta; i.e. to Clifford Phase Spaces. The gauge theory is associated to the fiber bundle over the base Clifford Phase-Space manifold with commuting polyvector-valued coordinates and momenta $X^{M}=x, x^{\mu}, x^{\mu \nu}, \ldots ; P^{M}=$ $p, p^{\mu}, p^{\mu \nu}, \ldots \ldots .$. The indices $\mu, \nu, .$. span $1,2,3,4$ and $1,2,3, \ldots \ldots, D$ in general. We denote the coordinates of the Clifford Phase-Space collectively by $Z^{M}=\left(X^{M}, P^{M}\right)$.

The polyvector extension of the Weyl-Heisenberg algebra involves the generators

$Y_{A}=\frac{1}{\sqrt{2}}\left(\frac{X_{A}}{\lambda_{l}^{|A|}}-i \frac{P_{A}}{\lambda_{p}^{|A|}}\right) ; \quad \bar{Y}_{A}=\frac{1}{\sqrt{2}}\left(\frac{X_{A}}{\lambda_{l}^{|A|}}+i \frac{P_{A}}{\lambda_{p}^{|A|}}\right) ; \quad A=1,2,3, \ldots \ldots . ., 2^{D}$

where $\lambda_{l}, \lambda_{p}$ are the Planck length and Planck momentum which can be set to unity when one chooses the natural units $\hbar=c=G=b=1$. $|A|$ denotes the grade of the polyvector index. The generators in (3.18) are chosen to be dimensionless.

Notice that we must not confuse the generators $X_{A}, P_{A}$ (associated with the fiber coordinates of the internal space of the fiber bundle) with the base manifold polyvector-valued coordinates and momenta $X^{M}, P^{M}$ corresponding to the Clifford Phase-Space. One may notice that the interval (2.8) in the base Clifford Phase-Space manifold requires the introduction of the upper Hubble and lower Planck scale, in addition to the maximal force $\mathcal{F}$ given by eq- $(2.9)$. Where the $\lambda_{l}, \lambda_{p}$ length and momentum scales in (3.18) are introduced in the expression of the polyvector extension of the Weyl-Heisenberg algebra generators in order to render them dimensionless. The extended Weyl-Heisenberg algebra is

$\left[X_{A}, P_{B}\right]=\left[X_{a_{1} a_{2} \ldots \ldots a_{n}}, P_{b_{1} b_{2} \ldots \ldots b_{n}}\right]=i \eta_{A B} \mathcal{I}=i \eta_{\left[a_{1} a_{2} \ldots \ldots a_{n}\right]\left[b_{1} b_{2} \ldots . . b_{n}\right]} \mathcal{I}$

The nonvanishing elements belong to the same-grade components of the fiber space metric $\eta_{A B}=\eta_{a_{1} a_{2} \ldots a_{n}} b_{1} b_{2} \ldots b_{n}$ and which can be decomposed into its irreducible factors as antisymmetrized sums of products of $\eta_{a b}$ as follows

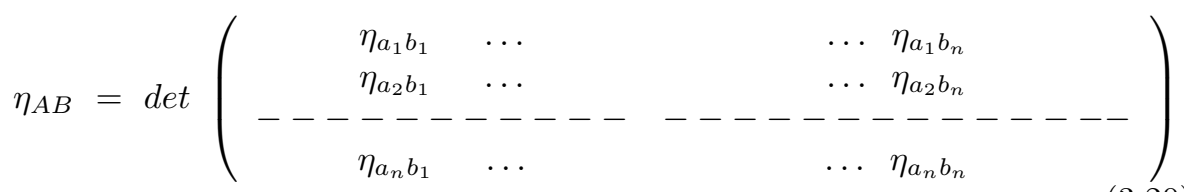

The mixed grade metric components $\eta_{A B}$ are zero. 
The generators $Y_{A B}$ can be decomposed into the "Lorentz" like generators $\mathcal{L}_{[A B]}$ and the "shear"-like generators $\mathcal{M}_{(A B)}$ as

$$
Y_{A B} \equiv \frac{1}{2}\left(\mathcal{M}_{A B}-i \mathcal{L}_{A B}\right) ;
$$

and the explicit commutation relations of the above generators are

$$
\begin{gathered}
{\left[\mathcal{L}_{A B}, \mathcal{L}_{C D}\right]=\left(\eta_{B C} \mathcal{L}_{A D}-\eta_{A C} \mathcal{L}_{B D}-\eta_{B D} \mathcal{L}_{A C}+\eta_{A D} \mathcal{L}_{B C}\right) .} \\
{\left[\mathcal{M}_{A B}, \mathcal{M}_{C D}\right]=-\left(\eta_{B C} \mathcal{L}_{A D}+\eta_{A C} \mathcal{L}_{B D}+\eta_{B D} \mathcal{L}_{A C}+\eta_{A D} \mathcal{L}_{B C}\right) .} \\
{\left[\mathcal{L}_{A B}, \mathcal{M}_{C D}\right]=\left(\eta_{B C} \mathcal{M}_{A D}-\eta_{A C} \mathcal{M}_{B D}+\eta_{B D} \mathcal{M}_{A C}-\eta_{A D} \mathcal{M}_{B C}\right) .}
\end{gathered}
$$

The commutators of $\mathcal{L}_{A B}$ with the $X_{C}, P_{C}$ generators are

$\left[\mathcal{L}_{A B}, X_{C}\right]=\left(\eta_{B C} X_{A}-\eta_{A C} X_{B}\right) ; \quad\left[\mathcal{L}_{A B}, P_{C}\right]=\left(\eta_{B C} P_{A}-\eta_{A C} P_{B}\right)$.

The commutators of $M_{A B}$ with the $X_{C}, P_{C}$ generators are

$\left[\mathcal{M}_{A B}, X_{C}\right]=-i\left(\eta_{B C} P_{A}+\eta_{A C} P_{B}\right) ; \quad\left[\mathcal{M}_{A B}, P_{C}\right]=-i\left(\eta_{B C} X_{A}+\eta_{A C} X_{b}\right)$

The gauge field $\mathbf{A}_{M}$ is given in terms of the connection, frame field as

$$
\mathbf{A}_{M}=\Omega_{M}^{A B} Y_{A B}+i\left(E_{M}^{A} Y_{A}+\bar{E}_{M}^{A} \bar{Y}_{A}\right)+i \Omega_{M} \mathcal{I} .
$$

where the complex frame $E_{M}^{A}$ which is no longer a square matrix is defined as

$$
E_{M}^{A}=\frac{1}{\sqrt{2}}\left(e_{M}^{A}+i f_{M}^{A}\right) ; \quad \bar{E}_{M}^{A}=\frac{1}{\sqrt{2}}\left(e_{M}^{A}-i f_{M}^{A}\right) .
$$

The complex Hermitian metric is given by

$$
G_{M N}=\bar{E}_{M}^{A} E_{N}^{B} \eta_{A B}=g_{(M N)}+i g_{[M N]} \equiv g_{(M N)}+i B_{M N} .
$$

such that

$$
\left(G_{M N}\right)^{\dagger}=\bar{G}_{N M}=G_{M N} ; \quad \bar{G}_{M N}=G_{N M} .
$$

where the bar denotes complex conjugation. Despite that the metric is complex the infinitesimal line element is real

$$
d s^{2}=G_{M N} d Z^{M} d Z^{N}=g_{(M N)} d Z^{M} d Z^{N} \text {, because } i g_{[M N]} d Z^{M} d Z^{N}=0 \text {. }
$$

The connection $\Omega_{M}^{A B}$ in (3.26) can be decomposed into anti-symmetric $[A B]$ and symmetric $(A B)$ pieces with respect to the internal indices

$$
\Omega_{M}^{A B}=\Omega_{M}^{[A B]}+i \Omega_{M}^{(A B)}
$$


giving

$$
\Omega_{M}^{A B} Y_{A B}=-\frac{i}{2} \Omega_{M}^{[A B]} \mathcal{L}_{A B}+\frac{i}{2} \Omega_{M}^{(A B)} \mathcal{M}_{A B}
$$

Given $Z^{\hat{M}}=\left(X^{M}, P^{M}\right), \partial_{\hat{M}}=\left(\partial / \partial X^{M}, \partial / \partial P^{M}\right)$, dropping the hats in the indices $Z^{\hat{M}}$ for convenience, the field strength is defined as

$$
\begin{gathered}
\mathbf{F}_{M N}=\partial_{M} \mathbf{A}_{N}-\partial_{N} \mathbf{A}_{M}+\left[\mathbf{A}_{M}, \mathbf{A}_{N}\right]= \\
F_{M N}^{A B} Y_{A B}+i\left(F_{M N}^{A} Y_{A}+\bar{F}_{M N}^{A} \bar{Y}_{A}\right)+F_{M N} \mathcal{I}= \\
\frac{i}{2} F_{M N}^{(A B)} \mathcal{M}_{A B}-\frac{i}{2} F_{M N}^{[A B]} \mathcal{L}_{A B}+i\left(F_{M N}^{A} Y_{A}+\bar{F}_{M N}^{A} \bar{Y}_{A}\right)+F_{M N} \mathcal{I}
\end{gathered}
$$

after decomposing $Y_{A B}=\frac{1}{2}\left(\mathcal{M}_{A B}-i \mathcal{L}_{A B}\right)$. The components of the curvature two-form associated with the anti-Hermitian connection $\Omega_{M}^{A B}=\Omega_{M}^{[A B]}+i \Omega_{M}^{(A B)}$ are

$$
\begin{gathered}
-i F_{M N}^{[A B]}=\partial_{M} \Omega_{N}^{[A B]}-\partial_{N} \Omega_{M}^{[A B]}+\Omega_{[M}^{[A C]} \Omega_{N]}^{[C B]}-\Omega_{[M}^{(A C)} \Omega_{N]}^{(C B)} . \\
i F_{M N}^{(A B)}=\partial_{M} \Omega_{N}^{(A B)}-\partial_{N} \Omega_{M}^{(A B)}+\Omega_{[M}^{(A C)} \Omega_{N]}^{[C B]}+\Omega_{[M}^{(B C)} \Omega_{N]}^{[C A]}
\end{gathered}
$$

where a summation over the repeated $C$ indices is implied and $[M N]$ denotes the anti-symmetrization of indices with weight one.

The components of the torsion are

$$
\begin{aligned}
& F_{M N}^{A}=\partial_{M} E_{N}^{A}-\partial_{N} E_{M}^{A}-i \Omega_{[M}^{[A C]} E_{N]}^{C}+i \Omega_{[M}^{(A C)} \bar{E}_{N]}^{C}-2 i \bar{E}_{[M}^{A} \Omega_{N]} \\
& \bar{F}_{M N}^{A}=\partial_{M} \bar{E}_{N}^{A}-\partial_{N} \bar{E}_{M}^{A}+i \Omega_{[M}^{[A C]} \bar{E}_{N]}^{C}-i \Omega_{[M}^{(A C)} E_{N]}^{C}+2 i E_{[M}^{A} \Omega_{N]}
\end{aligned}
$$

The remaining field strength assoiated with the $\mathcal{I}$ generator is

$$
F_{M N}=i \partial_{M} \Omega_{N}-i \partial_{N} \Omega_{M}+E_{M}^{A} \bar{E}_{N}^{B} \eta_{A B}-\bar{E}_{M}^{A} E_{N}^{B} \eta_{A B}
$$

The curvature tensor is defined in terms of the field strength and complex frame fields as

$$
\mathcal{R}_{M N P}^{Q} \equiv \frac{1}{4}\left(F_{M N}^{[A B]}+i F_{M N}^{(A B)}\right)\left(E_{A}^{Q} E_{B P}+\bar{E}_{A}^{Q} \bar{E}_{B P}+E_{A}^{Q} \bar{E}_{B P}+\bar{E}_{A}^{Q} E_{B P}\right) .
$$

where the explicit components $F_{M N}^{[A B]}$ and $F_{M N}^{(A B)}$ can be read from the defining relations $(3.34,3.35)$. The contraction of indices yields two different complexvalued (Hermitian) Ricci tensors.

$$
\mathcal{R}_{M P}=g^{K N} g_{Q K} R_{M N P}^{Q}=\delta_{Q}^{N} R_{M N P}^{Q}=R_{(M P)}+i R_{[M P]} ; \quad\left(\mathcal{R}_{M P}\right)^{*}=\mathcal{R}_{P M}
$$


and

$$
\mathcal{S}_{M \lambda}=g^{K N} g_{K Q} R_{M N P}^{Q}=\mathcal{S}_{(M P)}+i \mathcal{S}_{[M P]} ; \quad\left(\mathcal{S}_{M P}\right)^{*}=\mathcal{S}_{P M}
$$

due to the fact that

$$
g^{K N} g_{Q K}=\delta_{Q}^{N} ; \quad g^{K N} g_{K Q} \neq \delta_{Q}^{N} .
$$

because $g_{K Q} \neq g_{Q K}$. The position of the indices is crucial. There is a third Ricci tensor $Q_{[M N]}=\mathcal{R}_{M N P}^{Q} \delta_{Q}^{P}$ related to the curl of the nonmetricity Weyl vector $Q_{M}$ which one may set to zero. However, in the most general case one should include nonmetricity.

A further contraction yields the generalized (real-valued) Ricci scalars

$$
\begin{gathered}
\mathcal{R}=\left(g^{(M P)}+i g^{[M P]}\right)\left(R_{(M P)}+i R_{[M P]}\right)= \\
g^{(M P)} R_{(M P)}-B^{M P} R_{[M P]} ; \quad g^{[M P]} \equiv B^{M P} \\
\mathcal{S}=\left(g^{(M P)}+i g^{[M P]}\right)\left(S_{(M P)}+i S_{[M P]}\right)= \\
g^{(M P)} \mathcal{S}_{(M P)}-B^{M P} \mathcal{S}_{[M P]} .
\end{gathered}
$$

We should notice that the inverse complex metric is

$$
g^{(M P)}+i g^{[M P]}=\left[g_{(M P)}+i g_{[M P]}\right]^{-1} \neq\left(g_{(M P)}\right)^{-1}+\left(i g_{[M P]}\right)^{-1} .
$$

so $g^{(M P)}$ is now a complicated expression of both $g_{(M P)}$ and $g_{[M P]}=B_{M P}$. The same occurs with $g^{[M P]}=B^{M P}$. Rigorously we should have used a different notation for the inverse metric $\tilde{g}^{(M P)}+i \tilde{B}^{[M P]}$, but for notational simplicity we chose to drop the tilde symbol.

One could add an extra contribution to the complex-gravity real-valued action stemming from the terms $i B^{M P} F_{M P}$ which is very reminiscent of the $B F$ terms in Schwarz Topological field theory and in Plebanksi's formulation of gravity. In the most general case, one must include both the contributions from the torsion and the $i B^{M P} F_{M P}$ terms. The contractions involving $G^{M P}=$ $g^{(M P)}+i B^{M P}$ with the components $F_{M P}$ (due to the antisymmetry property of $\left.F_{M P}=-F_{P M}\right)$ lead to

$$
\begin{aligned}
i B^{M P} F_{M P}= & -B^{M P}\left(\partial_{M} \Omega_{P}-\partial_{P} \Omega_{M}\right)-2 B^{M P} B_{M P}= \\
& -B^{M P} \Omega_{M P}-2 B^{M P} B_{M P} .
\end{aligned}
$$

These $B F$ terms contain a mass-like term for the $B_{M P}$ field. When the torsion is not constrained to vanish one must include those contributions as well. The real-valued torsion two-form is $\left(F_{M N}^{A} Y_{A}+\bar{F}_{M N}^{A} \bar{Y}_{A}\right) d Z^{M} \wedge d Z^{N}$ and the torsion tensor and torsion vector are

$$
T_{M N}^{P}=F_{M N}^{A} E_{A}^{P} ; \quad \bar{T}_{M N}^{P}=\bar{E}_{A}^{P} \bar{F}_{M N}^{A} ; \quad T_{M N P}=g_{P Q} T_{M N}^{Q} ;
$$


$\bar{T}_{M N P}=\bar{T}_{M N}^{Q}\left(g_{P Q}\right)^{*}=\bar{T}_{M N}^{Q} g_{Q P} ; \quad T_{M}=\delta_{P}^{N} T_{M N}^{P} ; \quad \bar{T}_{M}=\bar{T}_{M N}^{P} \delta_{P}^{N}$.

The (real-valued) Lagrangian density linear in the two (real-valued) Ricci curvature scalars and quadratic in the torsion is of the form

$$
\mathcal{L}=a_{1} \mathcal{R}+a_{2} \mathcal{S}+a_{3} T_{M N Q} T^{M N Q}+a_{4} T_{M} T^{M}+c . c
$$

where one must add the complex conjugate (cc) terms in order to have a realvalued action and $a_{1}, a_{2}, a_{3}, a_{4}$ are suitable numerical coefficients. The analog of Einstein's vacuum field equations without torsion are

$$
\begin{aligned}
& \left(\mathcal{R}_{(M N)}+\mathcal{S}_{(M N)}\right)-\frac{1}{2} g_{(M N)}(\mathcal{R}+\mathcal{S})=0 \\
& \left(\mathcal{R}_{[M N]}+\mathcal{S}_{[M N]}\right)-\frac{1}{2} g_{[M N]}(\mathcal{R}+\mathcal{S})=0
\end{aligned}
$$

To conclude, the extended Born Reciprocal Gravitational theory in Clifford Phase Spaces involving symmetric and antisymmetric metrics differs from the gravitational theories in the literature, like in [25], [27], [24]. Gravitational theories based on Born's reciprocal relativity principle involving a maximal speed limit and a maximal proper force is a very promising avenue to quantize gravity that does not rely in breaking the Lorentz symmetry at the Planck scale, in contrast to other approaches based on deformations of the Poincare algebra, Hopf algebras, quantum groups, etc... Polyvector-valued gauge field theories in Noncommutative Clifford spaces were studied in [29]. Such field theory is the proper arena to construct a Noncommutative Gravitational theory in Clifford Phase Spaces.

\section{Conclusion : Finsler Geometry and Upper/Lower Bounds to Higher Order Accelerations}

To finalize we must point out the importance of Finsler geometry [13] in constructing an extended relativity theory based on setting bounds to the higher order accelerations in addition to the velocity and ordinary acceleration. A thorough study of Finsler geometry and Clifford algebras has been undertaken by Vacaru [12] where Clifford/spinor structures were defined with respect to nonlinear connections associated with certain nonholonomic modifications of Riemann-Cartan gravity. The study of nonholonomic Clifford-Structures in the construction of a Noncommutative Riemann-Finsler Geometry can be found also in [12].

The fundamental problem in constructing an extended relativity theory based on setting bounds to the higher order accelerations is the following. Let 
us take the simplest extension where one wishes to impose bounds on the velocity, the first and second order accelerations. The generalization of the Born interval is

$$
(d s)^{2}=(d X)^{2}+(d P)^{2}+(d Q)^{2}
$$

omitting indices and numerical constants. $P$ is the momentum and $Q$ is the "momentum" of the momentum; i.e. it involves the second order acceleration. In order to build an extension of Born's reciprocal relativity we have to find a symmetry which leaves invariant the interval $(d s)^{2}$. It looks like a $S O(4+4+4)=$ $S O(12)$ algebra (ignoring signatures for the moment).

If one wishes to have an invariant triple wedge product

$$
d X \wedge d P \wedge d Q=\omega_{i j k} d X^{i} \wedge d P^{j} \wedge d Q^{k}
$$

under the extension of the Quaplectic group transformations one requires to introduce the (ternary) 3-ary "symplectic" transformations leaving invariant the tripe wedge product (4.2).

In Born's relativity we have the invariance group which is the intersection of $S O(4+4)$ and the ordinary symplectic group $S p(8)$. The intersection contains the unitary group $U(4)$ which allowed Low [10] to write down the symmetry transformations under velocity and acceleration boosts, etc .. One may ask the question is : what is the intersection of the group $S O(12)$ with the ternary group ( $S O(4 n)$ and $n$-ary group in general ) which leaves invariant the triplewedge product (4.2) and the interval (4.1) ? A careful study reveals that this is the wrong question. The correct way to proceed is the following. Instead of writing the quadratic form (4.1) one must begin with a cubic norm

$$
(d s)^{3}=d_{i j k} d X^{i} d P^{j} d Q^{k}
$$

where $d_{i j k}$ is a symmetric rank three tensor. A realm of mathematics where cubic norms are very relevant is in the construction of Jordan algebras. They have been classified by Schafer [30] and there are three cases. In particular the four "magical" cases consisting of $3 \times 3$ hermitain matrices whose components take values in the four division algebras, real, complex, quaternions and octonions. The four magical Jordan algebras were key ingredients in the construction of magical supergravities [31].

Now one can ask the proper question : what is the ternary algebra resulting from the intersection of the ternary algebras which leave invariant the 3 -form (4.2) and the cubic norm (4.3) ? . One can then extend this construction to the n-ary algebra case by having

$$
\begin{gathered}
(d s)^{n}=d_{i_{1} i_{2} \ldots i_{n}} d Z_{(1)}^{i_{1}} d Z_{(2)}^{i_{2}} \ldots \ldots . . d Z_{(n)}^{i_{n}} \\
\omega^{(n)}=\omega_{i_{1} i_{2} \ldots \ldots i_{n}} d Z_{(1)}^{i_{1}} \wedge d Z_{(2)}^{i_{2}} \wedge \ldots \ldots \wedge d Z_{(n)}^{i_{n}}
\end{gathered}
$$

and then finding the the intersection of the $n$-ary algebras which leave invariant the $n$-form (4.5) and the $n$-norm (4.4). 
This procedure will allow us to find the $n$-ary analog of the Quaplectic group transformations which will now mix the $X, P, Q, \ldots \ldots$. coordinates of the higher order tangent (cotangent) spaces in this extended relativity theory based on Born's reciprocal gravity and $n$-ary algebraic structures. Before answering this difficult question one should recur firstly to the geometry of Higher-Order Finsler Spaces [13] and see whether or not one can set bounds to the higher order accelerations, and secondly, to study the symmetry transformation laws. In the meantime we need to explore the large number of novel physical consequences of Born's Reciprocal Gravitational theory in Clifford Phase Spaces described in this work.

\section{Acknowledgements}

We thank M. Bowers for very kind assistance.

\section{References}

[1] L. Nottale, La Relativite dans tous ses Etats. (Hachette Lit. Paris 1999).

L. Nottale Fractal Spacetime and Microphysics, Towards Scale Relativity (World Scientific, Singapore, 1992).

L. Nottale, Scale Relativity and Fractal Spacetime, A New Approach to Unifying Relativity and Quantum Mechanics (Imperial College Press, 2011).

[2] C. Castro and M. Pavsic, Progress in Physics vol 1 (April 2005) 31.

C. Castro and M. Pavsic Phys. Letts B 559 (2003) 74.

C. Castro and M. Pavsic, Int. J. Theor. Phys 42 (2003) 1693.

[3] C. Castro, Foundations of Physics 35, no.6 (2005) 971.

C. Castro, Prog. in Phys. 1 (April 2005) 20.

[4] W. Pezzagia, "Physical Applications of a Generalized Geometric Calculus" arXiv.org: gr-qc/9710027.

W. Pezzaglia, "Dimensionally Democratic Calculus and Principles of Polydimensional Physics arXiv.org: gr-qc/9912025.

[5] M. Pavsic, Found. of Phys. 33 (2003) 1277.

[6] M. Pavsic, The Landscape of Theoretical Physics : A Global View, from point particles to the brane world and beyond, in search of a Unifying Principle, (Fundamental Theories of Physics, vol. 19, Kluwer Academic Publishers, Dordrecht, Boston, London, 2001).

[7] S. Ansoldi, A. Aurilia and E. Spallucci, Clas. Quan. Grav. 19 (2002) 3207. S. Ansoldi, A. Aurilia, C. Castro and E. Spallucci, Phys. Rev. D 64 $026003(2001)$ 
[8] M. Born, Proc. Royal Society A 165291 (1938).

M. Born, Rev. Mod. Physics 21463 ( 1949).

[9] E. Caianiello, Lett. Nuovo Cimento 3265 (1981).

[10] S. Low, Jour. Phys A 355711 (2002).

S. Low, J. Math. Phys. 382197 (1997).

[11] C.Castro, "Progress in Clifford Space Gravity" to appear in Advances in Applied Clifford Algebras.

C. Castro, Int. J. Theor. Physics 52, Issue 1 (2013) 28.

[12] S. Vacaru, S. Vacaru, P. Stavrinos, E. Gaburov and D. Gonta, Clifford and Riemann- Finsler Structures in Geometric Mechanics and Gravity (Balkan Press, 2006).

S. Vacaru, "Non-holonomic Clifford-Structures and Noncommutative Riemann-Finsler Geometry" arXiv.org : math.DG/0408121.

S. Vacaru, "(Non) Commutative Finsler Geometry from String/M Theory " arXiv.org: hep-th/0211068.

[13] R. Miron, "Lagrangian and Hamiltonian geometries. Applications to Analytical Mechanics" arXiv.org : 1203.4101;

R. Miron, The Geometry of Higher-Order Lagrange Spaces. Applications to Mechanics and Physics, (Kluwer Acad. Publ. FTPH no. 82, 1997);

R. Miron, The Geometry of Higher-Order Finsler Spaces, (Hadronic Press Inc., SUA, 1998);

R. Miron, D. Hrimiuc, H. Shimada and S. Sabau, The Geometry of Hamilton and Lagrange Spaces (Kluwer Academic Publishers, Dordrecht, Boston, 2001).

[14] G. Amelino-Camelia, Int. J. Mod. Phys D 11 (2002) 35.

J. Lukierski, A. Nowicki, H. Ruegg and V. Tolstoy, Phys. Letts B 264 (1991) 331.

[15] G. Amelino-Camelia, L. Freidel, J. Kowalski-Glikman, and Lee Smolin, "The principle of relative locality" arXiv.org : 1101.3297.

[16] H. de Bie and F. Sommen, "Hermite and Gegenbauer polynomials in superspace using Clifford analysis" arXiv : 0707.2863.

[17] Carlos Castro, Phys Letts B 668 (2008) 442.

[18] C. Castro, Int. J. of Mod. Phys A 26, no. 21 (2011) 3653.

C. Castro, Foundations of Physics, vol 42, no. 9 (2012) 1135.

[19] C. Castro, Int. J. Mod. Phys. A27 (2012) 1250069;

C. Castro, Found. Phys. 42, no.8 (2012) 1031. 
[20] S. Capozziello and M. De Laurentis, Found.Phys. 40 (2010) 867.

[21] S. Capozziello and M. De Laurentis, Int.J.Geom.Meth.Mod.Phys. 5 (2008) 765 .

[22] C. Castro, Int. J. Geom. Methods of Mod Phys 8, no.6, (2011) 1239.

C. Castro, Advances in Applied Clifford Algebras 22, no. 1 (2012), 1.

[23] C.N Yang, Phys. Rev 72 (1947) 874. Proceedings of the International Conference on Elementary Particles, (1965) Kyoto, pp. 322-323.

[24] A. Einstein, Ann. Math 46, 578 (1945). A. Einstein and E. Strauss, Ann. Math 47, 731 (1946).

[25] J. Moffat, J. Math. Phys 36, no. 10 (1995) 5897. J. Moffat and D. Boal, Phys. Rev D 11, 1375 (1975). J. Moffat, "Noncommutative Quantum Gravity" arXiv : hep-th/0007181.

[26] T. Damour, S. Deser and J. McCarthy, Phys. Rev. D 47 (1993) 1541.

[27] A. Chamseddine, Comm. Math. Phys 218, 283 (2001). " Gravity in Complex Hermitian Spacetime" arXiv : hep-th/0610099.

[28] P. Achieri, M. Dimitrijevic, F. Meyer and J. Wess, "Noncommutative Geometry and Gravity" [arXiv : hep-th/0510059] and references therein.

[29] C. Castro, J. Phys A : Math. Theor. vol 43 (2010) 365201

[30] R. Schafer, J. Math. Mech. 10, no. 1 (1969) 159.

[31] M. Gunaydin, G. Sierra and P. Townsend, Nucl. Phys. B 242 (1984) 244. 